\title{
Balkanologie
}

Balkanologie Revue d'études pluridisciplinaires

Vol. VIII, $n^{\circ} 1$ | 2004

Volume VIII Numéro 1

\section{La diplomatie préventive au Kosovo : retour sur un échec retentissant (1989-1998)}

Preventive diplomacy in Kosovo: 1989-1999

\section{Emeric Rogier}

\section{(2) OpenEdition}

\section{Journals}

Édition électronique

URL : http://journals.openedition.org/balkanologie/513

DOI : 10.4000/balkanologie. 513

ISSN : 1965-0582

Éditeur

Association française d'études sur les Balkans (Afebalk)

Édition imprimée

Date de publication : 1 juin 2004

ISSN : 1279-7952

Référence électronique

Emeric Rogier, «La diplomatie préventive au Kosovo : retour sur un échec retentissant (1989-1998)», Balkanologie [En ligne], Vol. VIII, nº 1 | 2004, mis en ligne le 21 janvier 2010, consulté le 17 décembre 2020. URL : http://journals.openedition.org/balkanologie/513 ; DOI : https://doi.org/10.4000/ balkanologie. 513 


\title{
LA DIPLOMATIE PRÉVENTIVE AU KOSOVO : RETOUR SUR UN ÉCHEC RETENTISSANT [1989-1998]
}

\author{
Emeric Rogier*
}

Avant de faire la une en 1999 pendant soixante-dix-huit jours d'affilée puis de constituer une épine inextirpable dans le pied des diplomates européens, le Kosovo constitua un banc d'essai de la notion de "diplomatie préventive ". Entendue comme l'intervention d'un tiers visant à éviter l'éclatement d'un conflit armé dans une situation donnée, cette notion fut remise au goût du jour au lendemain de la guerre froide par l'ancien Secrétaire général de l'ONU, Boutros Boutros-Ghali. Les propositions contenues à cet égard dans l'Agenda pour la Paix ${ }^{1}$ soulevèrent un intérêt certain si bien que la diplomatie préventive, rebaptisée " prévention des conflits " pour mieux embrasser l'ampleur de la tâche et la diversité des modalités d'action, devint pour quelques années le mot d'ordre des institutions internationales de coopération et de sécurité. Curieusement, mais non sans raisons, le grand public s'est toujours montré beaucoup plus réservé sur le sujet. La seule expression de "prévention des conflits " tend ainsi à susciter au mieux un soupir de compassion, au pire un sourire poli. Appliquée aux Balkans, cette problématique semble être la marque, chez celui qui l'examine, d'une folie douce ou d'un idéalisme benêt. Et pourtant, la diplomatie préventive non seulement existe, mais est devenue, comme indiqué cidessus, un thème récurrent de la diplomatie multilatérale. Dans les Balkans au demeurant, il ne s'agit en rien d'une lubie : la diplomatie préventive y a même pris des formes si variées et sollicité tant d'acteurs que contrairement aux appa-

\footnotetext{
'Chargé de recherche à l'Institut Clingendael (Conflict Research Unit), La Haye, Pays-Bas. (erogier@clingendale.nl)
}

${ }^{1}$ Boutros-Ghali (Boutros), Agenda pour la Paix, New York : Nations unies, 1992. 
rences, cette région fait office de laboratoire ${ }^{2}$. L'expérience, on en convient volontiers, n'a pas toujours été concluante. C'est une raison supplémentaire de s'y intéresser. Il ne paraît pas inutile, par exemple, de chercher à comprendre par quel processus succéda au déploiement en 1992 d'une douzaine d'observateurs en République fédérale de Yougoslavie le déversement sept ans plus tard de milliers de bombes sur ce même pays. Or, l'action préventive, c'est la loi du genre, est rétive à la publicité et les régions en paix, en règle générale, n'attirent guère la curiosité des médias. Ainsi, la littérature sur le Kosovo sera bientôt aussi abondante que les munitions larguées par l'OTAN, mais se concentre bien davantage sur cette phase ultime du conflit que sur les tentatives antérieures de prévention.

Cet article se propose donc de revenir sur cette période relativement moins connue dans le but de contribuer, autant que faire se peut, à la connaissance du conflit du Kosovo ainsi qu'à la compréhension de la diplomatie multilatérale contemporaine. On ne retracera pas ici les conditions historiques et politiques qui firent de la province une bombe à retardement connue de tous dès l'éclatement de la Yougoslavie en 1989-1990. L'objectif, en revanche, est d'analyser pour quelles raisons il ne s'avéra pas possible d'éviter ce conflit si prévisible. Comment expliquer qu'en dépit de la connaissance dont disposaient les diplomates et décideurs occidentaux des risques de conflit dans la province et de leur souci d'empêcher une nouvelle guerre susceptible d'embraser l'ensemble de la péninsule balkanique, la diplomatie préventive se solda malgré tout par un échec ? En matière de prévention, le temps constitue une donnée clé. Pour répondre à cette question, on a donc privilégié une approche chronologique - articulée autour du moment charnière que constitua la signature de l'accord de Dayton en fin d'année 1995 - qui permet de mieux reconstituer et restituer les perceptions des différents acteurs. La première partie porte ainsi, comme son titre l'indique, sur les efforts de prévention " en attendant Dayton "- et notamment sur le traitement de la question du Kosovo dans les différentes conférences diplomatiques réunies autour du dossier yougoslave ainsi que sur les activités de terrain de l'OSCE et de l'ONU. La seconde partie est consacrée à l'après-Dayton ou comment se créa un décalage fatal entre la dynamique conflictuelle à l'œuvre dans la province et la politique de l'autruche qui fut alors celle des gouvernements occidentaux. Enfin, la troisième partie confronte les recommendations formulées par un certain nombre d'experts et d'organisations non-gouvernementales et les mesures prises in fine par les décideurs occidentaux en guise de stratégie préventive pour le Kosovo.

\footnotetext{
${ }^{2}$ Présentée en 2001 à l'Institut Universitaire de Hautes Études Internationales de Genève, notre thèse de doctorat, dont est issu cet article, portait précisément sur La prévention multilatérale des conflits armés dans les Balkans (Kosovo, Sandjak, Voïvodine, Macédoine) 1991-2001. Intitulèe Sous le signe de Sisyphe, cette thèse est diffusée en ligne à l'adresse suivante : http://www.unige.ch/cyberdocuments/theses2001/RogierE/meta.htm.
} 


\section{EN ATTENDANT DAYTON}

Avant de figurer pour un temps sous les sunlights des médias, le Kosovo constitua une ombre portée sur les conférences diplomatiques qui accompagnèrent la dissolution de la fédération yougoslave. Pas plus que les autres minorités de l'ex-Yougoslavie, les Albanais ne furent autorisés à siéger aux conférences de La Haye (1991) et Londres (1992) mais la question du Kosovo affleura malgré tout dans les discussions - qu'elle contribua même, dans le premier cas, à faire échouer. Si le Président Milošević rejeta le plan proposé par Lord Carrington pour tenter, à l'automne 1991, d'organiser un divorce à l'amiable entre les républiques yougoslaves, c'est ainsi notamment parce que la première version de ce texte envisageait la restauration du statut d'autonomie des provinces du Kosovo et de Voïvodine (une clause par la suite supprimée). L'échec de la Conférence de La Haye consommé, les Albanais du Kosovo adressèrent, au nom de la « République du Kosovo ", une demande de reconnaissance internationale, arguant notamment que la province constituait une entité fédérale au même titre que les autres républiques yougoslaves. Mais la Commission d'arbitrage présidée par Robert Badinter ne répondit pas même à cette requête qu'elle considéra vraisemblablement, émanant d'une minorité nationale, comme irrecevable. Lors de la Conférence de Londres des 26-27 août 1992 réunie à l'initiative des Britanniques par la Communauté européenne alors que l'existence de camps de concentration venait d'être révélée en Bosnie, la question du Kosovo ne constituait pas davantage la priorité des gouvernements occidentaux. En conséquence, les délégués albanais furent tout juste admis à suivre les débats sur un écran de télévision mis à disposition dans une salle parallèle. A cette occasion néanmoins, Milan Panić, le Premier ministre modéré de la République fédérale de Yougoslavie (RFY) tout juste nommé par Slobodan Milošević afin de donner des gages au monde extérieur, manifesta un esprit d'ouverture prometteur sur la question du Kosovo et des minorités de Serbie. C'est ainsi par son entremise que purent être lancées en 1992-1993 deux initiatives préventives sous les auspices respectifs de la Conférence internationale sur l'ex-Yougoslavie (CIEX) et de l'Organisation pour la Sécurité et la Coopération en Europe (OSCE). Toutefois, le Premier ministre fédéral devait tôt subir les foudres de la classe politique serbe et la " fenêtre d'opportunité " qu'il avait ouverte se refermer aussitôt qu'il eut quitté ses fonctions. Dès lors, la situation au Kosovo ne devait plus être suivie que de très loin par un contingent de " casques bleus " postés à la frontière macédonienne.

\section{Le groupe spécial sur le Kosovo de la Conférence internationale sur l'ex-Yougoslavie}

Établie en septembre 1992 à Genève, la CIEX résulta de la Conférence de Londres d'août 1992. Placée sous le double patronage de l'Union européenne et 
des Nations unies, la CIEX constituait un forum permanent de négociations, institué jusqu'au règlement final de l'ensemble des questions liées à la dissolution de la Yougoslavie. Bien que ses activités les plus en vue - et les plus controversées - aient touché au conflit de Bosnie, six groupes de travail furent établis, parmi lesquels celui sur " les minorités et communautés ethniques et nationales " chargé de " recommander les initiatives à prendre pour résoudre les problèmes ethniques dans l'ancienne Yougoslavie $n^{3}$. Dans ce cadre, la situation du Kosovo (ainsi que celles du Sandjak, de la Voïvodine et de la Macédoine) fut examinée pratiquement pour la première fois depuis l'éclatement de la fédération et des contacts directs, bien que peu fructueux, furent instaurés entre les protagonistes. Dans la forme comme sur le fond, il s'agissait sans conteste d'un exercice de diplomatie préventive.

Le titre alambiqué conféré à ce groupe travail donne cependant la mesure des difficultés auxquelles son président, l'Ambassadeur allemand Geert Ahrens, devait faire face. Il provenait du fait que par-delà leurs différences de situation, certaines des populations concernées avaient pour point commun de récuser l'appellation, jugée désobligeante, de " minorité " - ainsi des Serbes de Croatie qui se réclamaient de la nation serbe, des Musulmans du Sandjak qui se rattachaient à leurs " co-nationaux " de Bosnie-Herzégovine et des Albanais du Kosovo qui formaient la majorité de la province. Pour épargner les sensibilités et garantir la participation des intéressés, la difficulté fut noyée dans une formule nébuleuse.

De plus, nul consensus n'existait entre Serbes et Albanais sur le cadre juridique au sein duquel devaient être explorées des solutions au problème du Kosovo. D'après les statuts de la CIEX, le droit à l'autodétermination pouvait impliquer des dispositions en faveur de la protection des minorités mais ne pouvait entraîner une modification des frontières existantes 4 . Or, si les bons offices de la Conférence s'inscrivaient dans le droit fil d'une stratégie d'internationalisation conçue comme un pendant à la résistance pacifique, les Albanais du Kosovo revendiquaient, en contradiction avec ces principes, leur indépendance complète. Le régime Milošević refusait quant à lui toute ingérence extérieure dans les questions de minorités si bien que la médiation du groupe de travail fut acceptée par le seul gouvernement fédéral (et non par la République de Serbie).

Initialement, les médiateurs de la CIEX parvinrent bien à susciter un semblant de négociations mais ce processus devait tourner court en ce qu'il tenait,

\footnotetext{
${ }^{3}$ Le mandat de ce groupe tient dans cette seule phrase citée ; il figure à l'article 4 (c) du Programme de travail adopté par la Conférence de Londres, LC/C4 (final) du 27 août 1992. Cf. Ramcharan (B. G.), ed., The International Conference on Former Yugoslavia - Official Papers, The Hague/Boston/London : Kluwer Law International, vol.1, 1997, p. 35 (ci-après ICFY Official Papers).

4 " Statement of Principles, The London Conference, LC/C2 (Final), 26 August 1992 ", in ICFY Official Papers, p. 33.
} 
côté yougoslave, principalement à l'initiative d'un Milan Panić bien esseulé dans son camp (au cours de l'automne 1992, l'intéressé survécut de justesse à trois motions de censure déposées par les députés socialistes et radicaux du Parlement fédéral). C'est lui-même du reste qui suggéra la marche à suivre pour la conduite des pourparlers. L'idée générale était de " travailler sur une base pragmatique pour tenter de réussir une percée dans un secteur important afin d'améliorer l'atmosphère politique des discussions sur des questions plus fondamentales au sujet desquelles les positions sont actuellement irréconciliables " 5 . Les représentants albanais convinrent de cette " double approche " et choisirent de commencer par l'éducation.

À cette époque en effet, en conséquence de la reprise en main de la province par Belgrade, la quasi-totalité des quelque 300 ooo élèves albanais régulièrement inscrits à l'école publique jusqu'en 1990 étaient passés dans le système d'enseignement parallèle mis en place à tous les niveaux par la LDK ${ }^{6}$. Ce système comptait également environ 6000 étudiants albanais, préparant dans la clandestinité des diplômes que le gouvernement serbe ne reconnaissait pas, comme il ne reconnaissait pas la scolarité suivie aux niveaux inférieurs. L'enseignement primaire étant toutefois obligatoire, il était dispensé avec l'approbation tacite du gouvernement dans des locaux scolaires - mais au prix de la ségrégation complète des écoliers serbes et albanais, occupant un étage différent ou séparés par un mur7. L'enseignement secondaire et supérieur était assuré quant à lui dans des locaux privés - régulièrement perquisitionnés par les forces de police pour y rechercher les diplômes estampillés "République du Kosovo ", procéder à quelques interrogatoires musclés ou menacer les participants de représailles. C'est dans ce contexte que se déroulèrent les pourparlers sur l'éducation.

Précédée d'importants préparatifs, une première réunion trilatérale se solda le 14 octobre 1992 par l'adoption d'une déclaration commune fixant les objectifs des négociations - " rétablir d'urgence des conditions de travail normales dans les écoles et les autres établissements d'enseignement " - et la méthode - " sans préjudice des positions des parties sur les questions politiques plus générales $n^{8}$. D. Ćosić et I. Rugova convinrent peu après que les quatre niveaux d'enseignement (pré-scolaire, élémentaire, secondaire et supérieur) étaient visés par cette déclaration. Toutefois, de sérieuses divergences subsis-

\footnotetext{
${ }^{5}$ Rapport du Secrétaire général sur la Conférence internationale sur l'ex-Yougoslavie, S/24795 du 11 novembre 1992, § 90 .

${ }^{6}$ Voir notamment le rapport soumis par Tadeusz Mazowiecki, Rapporteur spécial de la commission des droits de l'Homme, S/26765 du 20 novembre 1993, $\$ 200-205$.
}

${ }^{7}$ Igric (Gordana), " Education is the Key in Serb-Kosovar Negotiations ", Transition, 3 (4), 7 March 1997 , p. 20.

${ }^{8}$ Rapport S/24795 (art.cit.), \$ 90. 
taient sur les conditions dans lesquelles la réouverture des bâtiments devait s'opérer. Alors que la délégation albanaise demandait la réouverture immédiate et sans conditions de l'ensemble des établissements, Belgrade souhaitait procéder niveau par niveau et exigeait au préalable le règlement des questions connexes, telles que les programmes d'enseignement, la réintégration des enseignants, la reconnaissance éventuelle de la scolarité et des examens passés dans le système " parallèle ". En fait, les Albanais souhaitaient disposer des bâtiments et assurer l'éducation des leurs libérés des contraintes de l'illégalité, mais récusaient tout droit de regard à la Serbie dont ils ne reconnaissaient pas, en tout état de cause, la juridiction. Et le gouvernement serbe, à l'inverse, cherchait à exercer un contrôle sur chacun des aspects de la question.

En somme, les négociations se déroulaient " sans préjudice " des positions des parties, mais non sans arrière-pensées. Il advint ainsi rapidement que la " double approche " aboutissait à une impasse, que les " problèmes concrets " n'en étaient pas moins des problèmes politiques et qu'on ne pouvait donc faire durablement abstraction des "questions fondamentales " liées à la souveraineté contestée du Kosovo. Le groupe de travail tenta de sauver les négociations en les élargissant à d'autres secteurs, tels que la santé, les médias et l'économie. Cette proposition reçut l'approbation des délégués albanais et du gouvernement fédéral, lequel se disait même prêt à aborder la question du statut. Mais pour cause d'échéances électorales en Serbie, nulle discussion ne put être entamée avant la défaite électorale de Milan Panić en décembre 1992. Début 1993, la " fenêtre d'opportunité " s'était refermée. À la reprise des négociations, Belgrade rejeta les plans d'enseignement albanais au motif qu'ils préjugeaient de l'indépendance du Kosovo et refusa d'évoquer tout autre sujet que les problèmes pédagogiques ${ }^{9}$. Les autorités serbes firent de nouvelles propositions au sujet de la réintégration des enseignants et de la reconnaissance de la scolarité effectuée dans le système parallèle, mais qui impliquaient de façon au moins implicite la reconnaissance de la souveraineté de la Serbie sur le Kosovo. Les Albanais les repoussèrent donc en bloc. La LDK n'avait en fait jamais donné à la CIEX d'autre fonction que de définir une solution internationale pour le Kosovo, une solution qui avait pour seul nom celui d'indépendance. Au second semestre 1993, c'en était fini des bons offices du groupe spécial. Celui-ci maintenait des contacts avec chacun des deux camps, mais sans parvenir à relancer le processus de négociation. En septembre, le gouvernement serbe lui fit savoir que les questions relatives aux minorités relevaient exclusivement de ses affaires intérieures ${ }^{10}$.

9 Cf. " Yugoslavia, Statement on Negotiations on Education Issues in Kosovo and Metohija, 29 August 1994 n, in Weller (Marc), The Crisis in Kosovo 1989-1999, Cambridge : Documents \& Analysis Publishing Itd (International Documents and Analysis, Volume 1), 1999, pp. 92-93. Ce document dresse le bilan des négociations du point de vue serbe.

10 « Rapport du Secrétaire général sur la Conférence internationale sur l'ex-Yougoslavie ", S/1994/83 du 26 janvier 1994, $\$ 15$. 
Aucun terrain d'entente ne se dégagea donc, seule se manifesta une commune hostilité au compromis qui illustre les limites de l'approche adoptée par le groupe de travail. L'idée sous-jacente de " réussir une percée afin d'améliorer l'atmosphère politique "s'avère biaisée car ce sont moins les négociations qui peuvent modifier le contexte que l'évolution de la situation qui permet d'engager des négociations. Or, les médiateurs de la CIEX n'avaient aucune influence sur ce contexte. Les observateurs de l'OSCE n'en eurent guère davantage.

\section{La diplomatie de terrain de l'OSCE}

L'implication de l'Organisation pour la sécurité et la coopération en Europe (OSCE) dans la diplomatie préventive sur le territoire de l'exYougoslavie relève à la fois de l'improvisation et d'un mouvement de fond. Improvisation, parce que les " Missions de longue durée " (MLD) mises en place au Kosovo, au Sandjak et en Voïvodine à l'automne 1992 ne résultaient d'aucun mécanisme ou plan d'action préconçu, mais figuraient une réponse ad hoc et sans véritable précédent. En même temps, cette diplomatie de terrain est devenue une pratique courante de l'OSCE, corrélative de la transformation de la Conférence sur la sécurité et la coopération en Europe (CSCE) en Organisation. $\mathrm{Au}$ lendemain de l'effondrement du communisme, la CSCE accomplit en effet une mutation spectaculaire, caractérisée par un triple développement normatif (engagements et principes), institutionnel (organes de décision et secrétariat) et opérationnel (missions et activités de terrain) que paracheva formellement l'adoption en décembre 1994 du sigle " OSCE " (pour Organisation). Dans le même temps, l'OSCE tendit à se spécialiser dans le domaine de la prévention des conflits, ce dont témoigne l'établissement des MLD.

L'OSCE dut cependant engager une partie difficile avec les autorités de Belgrade afin d'obtenir l'établissement d'une présence permanente dans les trois régions problématiques de Serbie. Alors que le pouvoir serbe luttait d'arrache-pied contre toute forme d'intrusion, synonyme à ses yeux d'internationalisation, ce sont à nouveau les autorités fédérales qui permirent de sortir de l'impasse et consentirent - peu après la nomination de $M$. Panić et du Président de RFY, D. Ćosić - à baisser sans condition le pont-levis ${ }^{11}$. Les MLD reçurent pour mandat de favoriser le dialogue entre les autorités concernées et les représentants des minorités, de recueillir des informations relatives aux violations des libertés fondamentales et à l'inverse de fournir une expertise sur la législation applicable en matière de droits de l'Homme, de protection des

${ }^{11}$ Le mémorandum d'accord fut signé à Belgrade le 28 octobre 1992 (CSCE Communication, (332), 03/11/92). La Mission commença néanmoins de fonctionner le 8 septembre 1992, sur la base des assurances données par le Premier ministre fédéral. 
minorités, de liberté des médias et d'élections démocratiques ${ }^{12}$. Une claire corrélation était ainsi établie entre violations des droits de l'Homme et risques de conflit, qui conférait une autre dimension aux " problèmes ethniques " identifiés dans les statuts de la CIEX. En outre, contrairement au groupe travail dont elles devaient soutenir l'action, ces Missions n'étaient pas formellement mandatées pour engager des négociations sur le fond entre les protagonistes. En vertu de leur double fonction d'observation et d'assistance, leur travail consistait davantage à tenter d'améliorer le climat général qu'à parvenir à des solutions durables.

Les MLD disposaient d'un siège à Belgrade, mais opéraient depuis des délégations installées dans chacune des trois régions concernées. Initialement fixé à 12 personnes, leur effectif total autorisé fut porté à 40 mais en vérité, il n'excéda jamais les 20 membres. Leur implantation permanente dans la province permit toutefois aux observateurs de se familiariser assez rapidement avec le contexte, d'entretenir des contacts assidus avec les principaux acteurs et d'analyser avec une certaine acuité leurs stratégies politiques. À cet égard, la Mission au Kosovo entoura ses activités de suffisamment de précautions pour éviter les risques de manipulation tout en incitant les organisations albanaises de défense des droits de l'Homme à mener une action plus efficace ${ }^{13}$. Alors que celles-ci avaient pour souci principal d'accumuler les témoignages à charge contre la souveraineté serbe, la Mission les encouragea à étayer leurs allégations, à constituer des dossiers plus solides ainsi, in fine, qu'à recourir aux tribunaux. En certains cas, les autorités locales auraient prêté une oreille plus attentive que de coutume aux observations de la Mission et fini par admettre l'existence d'un vrai problème de protection des droits de l'Homme. Quelques officiels serbes gravitant dans les cercles gouvernementaux songèrent même que la Mission de l'OSCE aurait pu aider le pouvoir à sortir de l'impasse en jouant notamment de son influence auprès de représentants albanais modérés déplorant pour leur part l'intransigeance de la LDK. Les uns et les autres se trouvaient cependant bien isolés. Dans l'ensemble, la Mission de l'OSCE n'avait pas la moindre influence sur une police serbe intraitable, rétive à toute conciliation et répondant exclusivement de Belgrade. Alors que nulle amélioration tangible de la situation n'avait de chance de se produire tant que se poursuivraient perquisitions illégales, arrestations arbitraires et brutalités policières, le régime serbe ne percevait pas dans son intérêt d'améliorer la situation sur ce terrain, mais misait au contraire sur son pouvoir militaire pour entamer la détermination des Albanais et les obliger à renoncer. De leur côté, s'ils étaient

\footnotetext{
${ }^{12} \mathrm{Cf}$. Journal, (2), 14/08/92, $15^{\text {ème }}$ réunion du CHF (annexe 1).

${ }^{13}$ Ce passage s'appuie notamment sur les rapports de la Mission au Kosovo (en particulier le $"$ Special Report : Kosovo-Problems and Prospects " et le " Final Report ") dont M. Weller a publié de larges extraits dans le recueil de documents précité (Weller (Marc), op.cit., pp. 107-119).
} 
conscients que l'indépendance leur était pour l'heure refusée, les dirigeants albanais escomptaient que les atteintes aux droits de l'Homme fassent à terme pencher la balance. Dans l'impossibilité d'atteindre leur libération, ils n'en considéraient pas moins que le temps jouait en leur faveur et percevaient le déploiement des observateurs de l'OSCE comme un premier signe tangible du bien-fondé de leur stratégie d'internationalisation. Autrement dit, chacun des deux camps entendait l'emporter à l'usure. La Mission au Kosovo tenta bien de promouvoir une appréciation plus réaliste de la situation, mais essuya un refus poli des dirigeants albanais, qui ne lui donnaient d'autre fonction que celle de faire-valoir, et les critiques de plus en plus vives du Parlement serbe et des médias - lesquels auront le dernier mot.

La Mission de l'OSCE ne devait son existence qu'à l'acceptation des autorités fédérales avec lesquelles fut signé le mémorandum d'accord en précisant les modalités. Or, si Milošević se fit surprendre par les deux hommes de paille qu'il pensait avoir placés au sommet des autorités fédérales, le Président serbe reprit la situation en main et organisa la chute de ses rivaux dès lors que son pouvoir lui parut menacé. Milan Panić fut destitué par le parlement fédéral et regagna son Amérique d'adoption au lendemain de sa défaite aux élections présidentielles de décembre 1992. Bien que massivement soutenu par l'opposition, il souffrit à cette occasion d'une assise électorale par trop étroite - que l'abstention des Albanais ( $12 \%$ des électeurs de Serbie) n'aida pas à élargir. Refusant de cautionner les institutions serbes et fédérales, ces derniers boycottèrent en effet l'ensemble des scrutins organisés en RFY dans les années 1990. En outre, la LDK ne jugeait probablement pas dans son intérêt la victoire de Milan Panić car celui-ci s'engageait à " seulement " restaurer les droits de l'Homme au Kosovo. Non seulement, l'objectif de la LDK demeurait l'obtention de l'indépendance, mais cette cause aurait été plus difficile à défendre encore si la situation des droits de l'Homme s'était effectivement améliorée dans la province. En tout état de cause, l'hostilité des nationalistes serbes à l'encontre des MLD redoubla de violence dès la chute du gouvernement Panić. Une prorogation de mandat fut bien négociée en avril 1993 avec le Président Ćosić, qui offrit aux missions deux mois de sursis ${ }^{14}$. Mais avant même l'expiration du délai, Ćosić, qui se livra progressivement à de sévères critiques de Slobodan Milošević jusqu'à demander publiquement sa démission, fut destitué à son tour par les députés SPS et SRS du Parlement fédéral (31 mai). Le Président serbe reprit de la sorte le contrôle de la situation et interdit, une semaine plus tard, de proroger les visas des observateurs de l'OSCE.

Placée en porte-à-faux dès l'origine, la Mission se trouvait dans une position d'autant plus délicate qu'en juillet 1992, avant même son établissement, la 
Serbie-Monténégro avait été suspendue de l'OSCE. Si cette décision s'était inscrite dans un tout autre contexte - en considération des responsabilités imputées crescendo au pouvoir en place à Belgrade dans la guerre en Bosnie - elle n'était évidemment pas de nature à tempérer l'hostilité des autorités serbes vis-à-vis de la Mission. Celles-ci disposèrent par ailleurs d'un argument tout trouvé pour suspendre les MLD en exigeant la réintégration préalable de leur pays au sein de l'OSCE. Si donc l'affaire de la suspension servit de prétexte au régime serbe, qui dès l'origine avait réprouvé l'établissement des MLD et n'avait nullement l'intention de s'en embarrasser, l'OSCE ne s'en retrouva pas moins prise au piège et compromit durablement toute initiative préventive de sa part. C'est pour réclamer la réouverture des MLD qu'en août 1993 le Conseil de sécurité de l'ONU adopta, en vain, sa toute première résolution traitant de la situation au Kosovo15. Engagée dans un dialogue de sourds avec Belgrade, l'OSCE n'obtiendra jamais le retour de sa vingtaine d'observateurs. À l'automne 1998 , le régime serbe acceptera, sous peine de bombardements, qu'elle déploie 2000 " vérificateurs ", mais il ne s'agissait plus alors de diplomatie préventive. Dans l'intervalle, un Groupe informel ad hoc à composition ouverte fut établi à Vienne (rendant compte au Conseil permanent de l'OSCE) afin d'assurer le suivi de la situation. Mais à l'évidence, ce dispositif ne constituait qu'un modeste substitut aux MLD dont la présence sur le terrain permettait de collecter des informations de première main qui feraient défaut à l'avenir.

\section{Le déploiement préventif de l'ONU en Macédoine}

Tandis que le huis clos se refermait au Kosovo, des " casques bleus " se trouvaient déployés en Macédoine voisine le long de la frontière avec la Serbie et l'Albanie. Cette présence de l'ONU avait été sollicitée par le Président macédonien Kiro Gligorov en novembre 1992 et autorisée le mois suivant par le Conseil de Sécurité (résolution 795) ${ }^{16}$. Il s'agissait de la première opération préventive de l'histoire des Nations unies, c'est-à-dire antérieure à l'éclatement éventuel d'un conflit dans la zone de déploiement. En l'occurrence, les autorités macédoniennes redoutaient d'une part, les conséquences d'hostilités au Kosovo et d'autre part, une agression potentielle de la Serbie ${ }^{17}$.

À cette heure, la situation du Kosovo n'avait pas encore franchi le seuil du conflit armé et ne figurait donc qu'une menace virtuelle. Mais dans l'hypothèse où des combats éclateraient dans la province, Skopje craignait pour di-

\footnotetext{
${ }^{15}$ Résolution 855 du 9 août 1993. La seconde sera émise en mars 1998.

${ }^{16}$ D'un effectif autorisé d'un millier d'hommes (auxquels s'ajoutaient trente-cinq observateurs militaires et vingt-six policiers), ce contingent n'était initialement qu'un détachement de la Force de protection des Nations unies (FORPRONU) déployée en Croatie et en Bosnie.

17 " Rapport de la Mission de reconnaissance de la FORPRONU en Macédoine ", S/24923 du 9 décembre 1992 (annexe, §7-10).
} 
verses raisons que la Macédoine ne soit entraînée dans la tourmente : l'Albanie pourrait alors décider de prêter main forte aux combattants albanais en faisant transiter ses forces par la Macédoine, les Albanais de Macédoine pourraient eux aussi prendre part au conflit, et les zones majoritairement albanophones de Macédoine occidentale pourraient servir de base aux opérations albanaises au Kosovo. Sur ce scénario s'en greffait un second, celui d'une attaque de la Serbie à l'encontre de la Macédoine lancée soit pour débusquer l'ennemi albanais de ses bases arrières, soit pour assouvir une vieille ambition sur un territoire naguère appelé la "Serbie du Sud ". Et si, par bonheur, nulle opération militaire ne se déroulait en Macédoine, les autorités redoutaient encore " l'effet déstabilisant " pour le pays que provoquerait un éventuel afflux de réfugiés du Kosovo... En tout état de cause, ajoutaient-elles, la situation d'ensemble pourrait déboucher sur une guerre balkanique de vaste ampleur.

En fin de compte, deux mois à peine s'écoulèrent entre la demande officielle du Président Gligorov et l'arrivée des tout premiers " casques bleus ». Que ce détachement procédât d'une force déjà présente dans les républiques voisines avait il est vrai permis de réduire significativement les délais habituels de déploiement. Mais cette rapidité d'exécution tint également à la volonté d'agir du Conseil de sécurité. L'explosion successive de conflits en Slovénie, Croatie et Bosnie avait en effet donné corps à une théorie des dominos, surfaite mais saisissante, en vertu de laquelle la Macédoine pouvait constituer la quatrième - ou cinquième, selon la position occupée par le Kosovo - des " bombes à retardement " dont le territoire de l'ex-Yougoslavie semblait parsemé. Et le " scénario du pire ", évoqué par le Président Gligorov, selon lequel une déstabilisation de la Macédoine pourrait entraîner une guerre généralisée dans les Balkans mettant notamment aux prises la Grèce et la Turquie - deux membres de l'OTAN - convainquit les Occidentaux et particulièrement les États-Unis de stopper "l'extension du conflit yougoslave ".

Au regard de la gravité des périls dont la Macédoine et au-delà, la péninsule balkanique étaient dites menacées, les fonctions attribuées à la force de l'ONU étaient pourtant assez modestes. Il s'agissait d'une part, de constituer un système d'alerte précoce et d'autre part, d'exercer un effet dissuasif envers tout agresseur potentiel.

Les autorités de Skopje se sentaient en effet d'autant plus vulnérables qu'au cours de sa retraite (pourtant négociée avec le gouvernement macédonien) l'armée fédérale yougoslave (JNA) avait privé le nouvel État de la totalité de son armement lourd et de tout l'équipement nécessaire à la surveillance des frontières. Dans ces circonstances, le premier objectif du déploiement préventif était de remédier aux carences de l'État-hôte en installant du côté macédonien des frontières avec l'Albanie $(182 \mathrm{~km})$ et la République fédérale de Yougoslavie $(240 \mathrm{~km})$ un bataillon d'infanterie chargé de " surveiller l'évolution de la situation " et " signaler tout événement se produisant dans les zones 
frontalières qui pourrait saper la confiance et la stabilité en Macédoine ou menacer le territoire macédonien ${ }^{18}$. Furent ainsi progressivement installés une vingtaine de postes permanents d'observation auxquels s'ajoutèrent autant de postes temporaires.

La dimension dissuasive de la FORPRONU apparut plus nettement en juin 1993 après que les États-Unis eurent proposé d'adjoindre 300 de leurs soldats au bataillon nordique qui composait jusqu'alors la force. Entérinée par le Conseil de sécurité dans la résolution 842 du 18 juin 1993, la participation des États-Unis transformait l'assistance purement matérielle fournie par la FORPRONU en un soutien politique et devait faire clairement savoir, comme le souligna alors B. Boutros-Ghali, que "la communauté internationale n'accepter[ait] pas l'extension du conflit tragique dans cette région $»^{19}$. Par voie de conséquence, le détachement onusien n'avait plus pour seul objet d'avertir les autorités macédoniennes d'une agression imminente, mais bien d'avertir tout agresseur d'une réaction immédiate.

La décision américaine fut motivée par l'aggravation de la situation en Bosnie après l'échec du plan Vance-Owen et la crainte de voir le conflit se propager en direction du sud. Paradoxalement, les autorités macédoniennes ne s'inquiétaient pas à proprement parler de l'impact sur leur pays de la guerre faisant rage à l'époque en Bosnie - laquelle, faut-il le rappeler, n'est pas limitrophe de la Macédoine - mais des menaces provenant de la Serbie et du Kosovo. Non seulement donc l'hypothèse retenue par l'administration américaine était largement dénuée de fondements, mais la réalité était exactement l'inverse. En effet, le régime serbe, qui procéda au retrait des contingents de la JNA postés en Macédoine au titre des préparatifs de la guerre de Bosnie, ne risquait pas (si tant est qu'il en avait l'intention) de s'attaquer à la première tant qu'il était occupé avec la seconde et se gardait dans le même temps d'ouvrir un nouveau front au Kosovo. Ainsi, la poursuite de la guerre en Bosnie ne menaçait pas la Macédoine, mais au contraire lui accordait un sursis. Comme la suite le démontrera, c'est après la conclusion de l'Accord de Dayton qu'éclatera un nouveau conflit affectant, celui-ci, bien plus directement ce pays. La stratégie américaine d'endiguement était donc viciée ; elle n'avait par ailleurs guère d'impact sur la véritable bombe à retardement que constituait le Kosovo.

La présence de " casques bleus " à la lisière du Kosovo n'influait en effet en rien sur la résolution des problèmes qui se posaient dans cette région. En d'autres circonstances, le déploiement de la FORPRONU aurait pu revêtir une valeur dissuasive, s'il s'était agi de figurer une avant-garde prête à intervenir en cas d'offensive militaire de Belgrade dans la province. En décembre 1992, le

${ }^{18}$ Ibid., $\$ 3$.

19 " Rapport du Secrétaire général sur la FORPRONU ", S/26099 du 13 juillet 1993, § 24. 
Président Bush avait d'ailleurs lancé à Slobodan Milošević un avertissement en ce sens - ledit Christmas Warning réitéré par Bill Clinton après son élection. Les États-Unis se disaient alors prêts, en cas de conflit au Kosovo provoqué par une action militaire serbe, à employer la force contre les forces de Belgrade au sein de la province et de la Serbie proprement dite ${ }^{20}$. Toutefois, par-delà la posture prise par le gendarme américain, le Président serbe savait qu'aucun consensus n'existait entre les principales puissances pour recourir à la force contre son pays et plus encore, que celles-ci partageaient son opposition à l'indépendance du Kosovo (comme rappelé dans le programme d'action commun de mai 1993 par lequel Américains, Russes, Français, Britanniques et Espagnols enterrèrent le Vance-Owen). Le Président serbe savait également que, dépourvue du mandat, des moyens et de l'approbation des États fournisseurs de troupes - sans compter celle de l'État-hôte - la FORPRONU n'était aucunement destinée à se lancer, en cas de besoin, dans une opération d'imposition de la paix au Kosovo. Et la présence en son sein de soldats américains ne faisait pas la moindre différence, au contraire : conformément à la doctrine alors en vigueur du " zéro mort ", l'administration américaine n'envisageait pas de risquer la vie de ses GIs au cours d'une contre-offensive terrestre. Dans ces conditions, la FORPRONU ne jouait aucun rôle eu égard à la situation au Kosovo, ni celui de dissuader Belgrade de prendre une initiative militaire, ni non plus celui d'empêcher côté albanais d'éventuels trafics d'armes ou préparatifs par-delà les frontières (qu'elle ne contrôlait pas) du Kosovo, de l'Albanie et de la Macédoine. Sa seule fonction consistait, s'il y avait lieu, à signaler l'arrivée de réfugiés en provenance de la province. Au bout du compte, l'action entreprise par les " casques bleus " pour écarter la menace venue du nord était bien limitée au même titre du reste que l'ensemble des initiatives préventives prises en attendant Dayton.

\section{Bilan provisoire}

Dresser le bilan de toute initiative préventive, c'est répondre à deux questions : les risques de conflits qui motivèrent le lancement d'une telle action ont-ils été écartés ? Et dans l'affirmative, cette évolution incombe-t-elle aux efforts de prévention menés ? Lorsque la paix se profilera en BosnieHerzégovine, la tentation sera de répondre par l'affirmative à ces deux interrogations. L'Accord de Dayton mettant censément un terme aux hostilités en cours, le conflit " yougoslave " (au singulier) ne menace plus de s'étendre, ni ne

\footnotetext{
${ }^{20}$ L'administration Clinton réitéra cet avertissement en mars 1993 notamment, puis encore en août par la voix de $\mathrm{M}$. Albright, représentant alors les États-Unis à l'ONU : " Le message du Président Bush a été clair et précis : nous sommes prêts à réagir contre les Serbes dans l'éventualité d'un conflit au Kosovo provoqué par une action serbe. Le Secrétaire d'État Christopher a vigoureusement réaffirmé cette position n déclarait-elle après l'adoption de la résolution du Conseil de sécurité demandant le rétablissement des MLD (S/PV.3262 du 9 août 1993, p. 17).
} 
s'est propagé par la grâce des mesures prophylactiques antérieurement prises. La logique semble imparable : si l'objectif est atteint, c'est que les moyens étaient appropriés. Or, la réalité était toute contraire.

Les deux principales initiatives préventives dédiées au Kosovo lancées en attendant Dayton le furent au cours de " l'interlude Panić ". Le groupe de travail de la CIEX et les missions de l'OSCE avaient pour intérêt respectif de promouvoir un processus de négociations et d'instituer une présence sur le terrain. Mais ces deux initiatives s'effectuaient sans carotte ni bâton et reposaient exclusivement sur le bon vouloir des intéressés. Les négociateurs internationaux escomptaient de fait beaucoup du tandem, pourtant improbable, constitué par Dobrica Ćosić et Milan Panić. En fin de compte, ces initiatives furent torpillées par le régime serbe et servirent tout juste d'alibi aux Occidentaux, au même titre que Milan Panić lui-même. À compter de la mi1993, la diplomatie préventive en RFY se réduisit à une peau de chagrin. Le peu qui avait été fait jusqu'alors relevait d'une approche purement coopérative dont les échecs attestaient, dans ce cas précis, de l'inanité. Certes, en décembre 1992, l'administration américaine avait lancé son fameux Christmas Warning. Mais il s'agissait d'une démarche unilatérale - non d'une position collective avalisée, le cas échéant, par le Conseil de sécurité - qui masquait mal la réticence des États-Unis à envoyer leurs soldats au feu dans les Balkans. En outre, cette initiative n'exigeait pas du régime serbe qu'il lève l'état de siège au Kosovo ni qu'il engage des négociations. Enfin, en dépit de la participation des États-Unis, la FORPRONU n'en imposait guère et relevait plus de la mission d'observation. Paradoxalement, ce déploiement de troupes fut motivé par la crainte dénuée de fondements de voir le conflit bosniaque se " propager " (d'un bond ?) en Macédoine alors qu'à l'inverse, il ne permettait en rien de désamorcer la véritable bombe à retardement que constituait alors le Kosovo.

Si le conflit n'avait pas encore explosé dans la province, ce pouvait donc difficilement être à porter au crédit de la diplomatie préventive mais tenait plutôt à des facteurs intrinsèques. En fait, la situation au Kosovo dans les années 1992-1995 était à la fois stable et explosive. Stable dans la mesure où ni les autorités serbes, ni les dirigeants albanais ne considéraient alors dans leur intérêt de franchir le pas de la confrontation militaire. Les premières étaient engagées sur d'autres théâtres d'opérations, subissaient le poids croissant des sanctions internationales et redoutaient qu'une répression plus vigoureuse encore ne suscite la rébellion de la population albanaise. C'est ainsi, par exemple, que Belgrade se garda d'arrêter les dirigeants de la LDK en général et Ibrahim Rugova en particulier, lequel fut laissé libre de plaider la cause des Albanais à travers le monde. Bien conscients que l'issue pourrait être fatale à leur communauté, les seconds ne souhaitaient pas davantage enclencher le mécanisme de l'escalade, mais misaient sur le temps et l'implication interna- 
tionale. En somme, si la confrontation armée avait jusqu'alors été évitée, c'est qu'en comparaison des bénéfices escomptés, elle présentait un coût, politique pour les uns et humain pour les autres, trop élevé. La situation n'en demeurait pas moins explosive, car le fossé se creusait chaque jour un peu plus entre deux camps parfaitement réfractaires l'un à l'autre et fonctionnant en tous domaines (politique, économie, éducation, santé) de façon toujours plus séparée. Au fil des ans, le gouffre s'approfondit entre Serbes et Albanais, qui ne partageaient plus guère que suspicion mutuelle et inimitié réciproque. Au moment de son départ en 1993, la Mission de l'OSCE avait du reste souligné que les hostilités pourraient éclater au Kosovo après le règlement du conflit de Bosnie, si Belgrade décidait alors d'en finir, si l'extrême-droite serbe se livrait à des provocations ou bien encore, si des groupes albanais lassés de la résistance pacifique enclenchaient l'escalade ${ }^{21}$... À la faveur de Dayton précisément, la diplomatie préventive ne parut pourtant plus autant d'actualité.

\section{DAYTON OU LA PAIX EN TROMPE L'CEIL}

Le paradoxe de la diplomatie préventive dans les Balkans au cours de la décennie 1990 est que celle-ci fut déployée avec une intensité inversement proportionnelle à la probabilité des risques. Avant Dayton, il s'agissait pour l'essentiel d'éviter l'extension des hostilités à d'autres régions d'exYougoslavie, alors même que leur continuation agissait comme un frein à l'éclatement de nouveaux conflits au Kosovo et en Macédoine. Après Dayton, la prévention sembla perdre en pertinence, et perdit effectivement en intensité, cependant qu'augmentaient les risques d'affrontement dans certaines des régions auparavant épargnées.

\section{Les illusions de la paix}

Après trois semaines de négociations à huis clos, la Bosnie émerge de Dayton avec un nouveau visage, marqué des cicatrices de la guerre. L'ancienne république yougoslave demeure, au même titre que la Slovénie, la Croatie et la Macédoine, un État souverain et indépendant reconnu dans ses frontières internationales d'avant-guerre. De ce point de vue, les apparences sont sauves. Nulle modification par la force des frontières issues du processus de dissolution de la Yougoslavie ne fut entérinée, qu'il s'agisse de celles de la Croatie ou de la Bosnie. En revanche, celle-ci se trouve désormais divisée en deux entités sub-étatiques, la Fédération croato-musulmane et la Republika Srpska, séparées par une ligne de démarcation. Et dans les faits, la Bosnie est

${ }^{21}$ La Mission évoquait également l'hypothèse que la LDK renonce au pacifisme. Cf. " Final Report " (art.cit.). 
morcelée non pas en deux mais en trois territoires très largement homogènes, taillés chacun dans la chair et dans le sang au prix du déplacement forcé de la moitié, au moins, des habitants du pays. Si donc aucun changement des frontières externes ne fut accepté, l'Accord de Dayton entérine la création par la force de nouvelles délimitations internes et la constitution de territoires mono-communautaires. Sauf retour - sinon improbable, du moins laborieux - des réfugiés et personnes déplacées dans leurs foyers, le nettoyage ethnique aura fait son œuvre. Et dans tous les cas, à travers la reconnaissance de la Republika Srspka, démonstration fut apportée qu'une telle politique peut payer, légitimité fut donnée à l'appropriation au bénéfice exclusif d'une communauté d'un territoire en comptant plusieurs. Ce n'est plus seulement la nation, mais l'espace qui est ainsi défini en termes " ethniques " et se trouve dédié à une population spécifique.

En outre, si la plupart des différends opposant la Bosnie, la Croatie et la RFY sont en voie de résolution, demeurent pleines et entières les situations potentiellement conflictuelles sur le reste du territoire de l'ex-Yougoslavie et notamment au Kosovo. Comme son nom l'indique, l'Accord-cadre général pour la paix en Bosnie-Herzégovine ne scelle le règlement que de ce seul conflit (et ne contient, contrairement à une opinion largement répandue à l'époque, aucune allusion au Kosovo). Alors par exemple que la Conférence de La Haye tendait à un règlement global de la dissolution yougoslave, les négociations conduites à Dayton même ou en parallèle ne portèrent que sur celui des conflits ouverts, avec les seules parties directement impliquées ${ }^{22}$. Mais précisément parce que le plan Carrington avait échoué sur la question des minorités, les médiateurs de Dayton craignirent de s'aliéner la coopération du Président serbe et de compromettre la conclusion d'un accord sur la Bosnie en soulevant d'autres sujets sensibles. Cependant, de même que la suspension des hostilités en Croatie s'accomplit au prix de leur éclatement en Bosnie, le règlement séparé du conflit dans cette république devait préluder à l'éclatement de celui du Kosovo. Pour trois raisons au moins : après La Haye et Londres, les Albanais de la province se voient une nouvelle fois sacrifiés au nom d'intérêts supérieurs, abandonnés à leur sort et à la poigne d'un régime désormais loué pour sa contribution à la paix dans la région; pour un certain nombre d'entre eux, Dayton légitime le recours à la force comme le moyen de se faire entendre et d'obtenir gain(s) de cause ; garant de l'Accord de paix, Slobodan Milošević tient en dépendance les Occidentaux qui souhaitent conserver son concours pour consolider le fragile édifice tout juste élaboré. Dayton est ainsi une paix en trompe l'oeil, qui marque certes l'achèvement de deux guerres effroyables

${ }^{22}$ La seule exception fut le règlement provisoire du différend gréco-macédonien obtenu en septembre 1995 en marge des préparatifs de Dayton avec un coup de pouce de Richard Holbrooke. 
dans la région, mais comporte les germes de conflits futurs ${ }^{23}$. Et pourtant, l'optimisme prévaut.

Comment l'expliquer ? Comment expliquer que diplomates et stratèges occidentaux prêtent alors si peu d'attention au Kosovo et ne prennent la mesure d'un danger si nettement décrit par nombre d'observateurs ? La première raison est que les Occidentaux se focalisent sur leur sujet de prédilection, la Bosnie cette " petite Yougoslavie " dont le destin semble devoir guider celui de l'exYougoslavie tout entière - et sur le calendrier d'application de l'accord de Dayton. À cet égard, que l'organisation des élections générales de septembre 1996 en Bosnie servit de critère au retrait de la force de l'ONU en Macédoine en dit long. Deuxième facteur d'inattention, la perspective d'une guerre balkanique, qui inspira la plupart des initiatives préventives avant Dayton, semble s'estomper pour de bon. La paix est rétablie en Bosnie et en Croatie et la guerre ne semble plus menacer aux portes de la Macédoine, après la normalisation intervenue au cours de l'année 1996 avec Athènes et Belgrade. Il demeure bien quelques zones d'ombre au tableau, et notamment la situation trouble au Kosovo. Toutefois, à la grande satisfaction des Occidentaux qui prétendent le soutenir en le discréditant, Ibrahim Rugova demeure attaché à la non-violence tandis que ses adversaires les plus virulents n'appellent pas (encore) aux armes. L'UÇK pour ce qui la concerne n'est alors qu'un mouvement fantomatique, aux yeux de la plupart des Albanais eux-mêmes. Par ailleurs, les Occidentaux ne croient pas que Slobodan Milošević, qui fournit tant d'efforts pour se refaire une virginité, soit prêt à se lancer dans une nouvelle expédition militaire. Or, suite à l'invalidation des élections municipales de novembre 1996 remportées par l'opposition, le nouveau Président de RFY (élu à ce poste qu'il était seul à briguer en juillet 1996 par le parlement fédéral) devait affronter en Serbie même une vague de contestation comme il n'en avait jamais connu. De cette épreuve et d'autres déboires électoraux, Slobodan Milošević conclut ainsi que la paix, en vérité, ne lui réussissait pas et que contrairement à ses calculs, la population ne lui en savait gré mais aspirait au changement. Douze mois après Dayton, resurgit ainsi un " besoin de guerre " pour faire diversion, étouffer la contestation et renouer avec l'exaltation national-communiste d'antan.

En définitive, les Occidentaux n'imaginaient pas que la situation au Kosovo puisse se dégrader aussi rapidement, ni qu'un nouveau conflit puisse surgir quelques mois seulement après qu'un terme fut mis au cauchemar de la Bosnie. Ils se l'imaginaient d'autant moins qu'à leurs yeux, chaque entité issue de l'exYougoslavie avait désormais trouvé sa place et son rang, la Bosnie, la Croatie, la Macédoine mais aussi le Kosovo qui devait demeurer au sein des frontières de

\footnotetext{
${ }^{23}$ Ce n'est pas dire toutefois que l'Accord de Dayton compte parmi les causes premières ou directes du conflit du Kosovo. Celui-ci couvait bien avant la cessation des hostilités en Bosnie-Herzégovine et puisait à des sources endogènes à la province. Dayton servit plus exactement de "catalyseur ".
} 
la RFY. Bref, ils s'accrochaient à l'idée, par eux-mêmes générée, que Dayton marquait un règlement à la fois définitif et régional du " conflit yougoslave ". La politique de l'autruche qui fut alors menée donne la mesure de leurs illusions.

\section{La politique de l'autruche}

Alors même que les périls montaient au Kosovo, les rares initiatives prises au cours de l'année 1996 s'avérèrent au mieux insignifiantes, au pire aggravantes. La façon dont le groupe de travail de Genève tomba progressivement en déshérence est assez révélatrice de la passivité générale. La Conférence de mise en œuvre de la paix organisée à Londres en décembre 1995 avait prononcé la dissolution de la CIEX, mais prorogé " aussi longtemps que nécessaire " le mandat de trois de ses groupes de travail - parmi lesquels celui dédié aux " questions ethniques " - qui furent transférés au Conseil de mise en œuvre de la paix (PIC) et, pour des raisons pratiques, rattachés au Bureau de Bruxelles du Haut Représentant (OHR) chargé de superviser l'application des aspects civils de l'Accord de Dayton ${ }^{24}$. Formellement, mais aussi politiquement, les activités de ce groupe étaient ainsi liées à la consolidation de la paix en Bosnie, au grand bénéfice de Slobodan Milošević. Quelle influence le premier Haut Représentant, Carl Bildt, pouvait-il exercer sur le Président de RFY à propos du Kosovo, alors qu'il avait tant besoin de sa coopération - et la sollicitait outrageusement selon certains - pour renforcer la paix en Bosnie ? À peine eut-il entrepris de modifier l'intitulé du groupe de travail « sur les communautés et minorités ethniques et nationales " en groupe de travail " sur les questions régionales " que les autorités fédérales, prétextant d'un changement implicite de mandat, menacèrent de suspendre leur participation ${ }^{25}$. Succédant à Geert Ahrens en mars 1996, l'Ambassadeur allemand Martin Lutz se vit ainsi initialement refuser un visa pour la RFY, au motif éculé mais toujours efficace de la non-ingérence. Au bout du compte, le groupe ne parvint pas à susciter le moindre échange à propos du Kosovo, sans toutefois que le PIC ne s'en émeuve outre mesure.

En 1996, l'heure est au bilan des activités préventives, un bilan dressé avec autant de subjectivité et de complaisance que l'analyse de la situation. Ainsi, le déploiement préventif de l'ONU fut qualifié de success story parce qu'il - ou bien qu'il - ne servit à rien et son retrait, un temps ajourné pour cause d'élec-

\footnotetext{
24 Cf. "Conclusions of the Peace Implementation Conference ", London : Lancaster House, 8-9 December 1995, $\$ 21$ (document S/1995/1029 du 12 décembre 1995) et Troebst (Stefan), Conflict in Kosovo: Failure of Prevention ? An Analytical Documentation, 1992-1998, London : ECMI Working Paper \# 1, May 1998, p. 39. Le PIC réunit la totalité des États, organisations et agences précédemment membres de la CIEX.
}

25 Troebst (Stefan), op.cit., p. 39. 
tions en Bosnie (!), fut corrélativement amorcé en $1997^{26}$. À l'évidence, la reconstruction de la Bosnie, aussi difficile fut-elle, n'avait guère plus d'impact sur la Macédoine que n'en eut sa destruction. En outre, l'éventualité d'une agression directe de la Serbie devint quasi-nulle à l'issue de l'accord de normalisation signé en avril 1996. En revanche, le problème du Kosovo restait entier et la probabilité qu'il ne dégénérât, et se répercutât d'une façon ou d'une autre en Macédoine, s'était même accrue depuis Dayton. Dans ces circonstances, ce n'est pas des modalités d'interruption ou de réduction de la FORDEPRENU dont le Conseil de sécurité aurait dû débattre, mais de son renforcement. Ce sont les moyens d'exercer une dissuasion sur le régime serbe, d'éviter la formation d'un mouvement armé parmi les Albanais, de contrôler les frontières, de restaurer les contacts politiques qui auraient dû susciter son attention. Il n'en fut rien. Conformément aux instructions reçues, les " casques bleus " commencèrent de plier bagage... au moment précis où la crise en Albanie les obligea à regagner d'urgence leurs postes d'observation. Au bout du compte, par la faute de la Chine qui refusa de proroger son mandat en représailles à la reconnaissance de Taiwan par le gouvernement macédonien, la FORDEPRENU quitta effectivement la Macédoine en février 1999... un mois avant que ne déferlent plus de 350000 réfugiés albanais dans le pays. Ce " premier déploiement préventif de l'histoire des Nations unies " s'acheva ainsi en queue de poisson, au moment même où se réalisait le scénario - ou certains de ses aspects - qui en avait motivé, six ans plus tôt, la mise en place ${ }^{27}$.

La seule mesure préventive adoptée à l'égard du Kosovo dans l'aprèsDayton fut en fait le " mur extérieur " de sanctions, lequel n'engageait toutefois que les États-Unis. Prenant acte de la conclusion de l'Accord de Dayton, le Conseil de sécurité avait décidé de suspendre les sanctions décrétées au long du conflit de Bosnie à l'encontre de la RFY et des autorités serbes de Pale (résolution 1022 du 22 novembre 1995) puis de les lever définitivement après la tenue dans les délais prévus des élections générales en Bosnie (résolution 1074 du $1^{\mathrm{er}}$ octobre 1996). S'il se réservait la possibilité de revenir sur cette décision en cas d'obstruction à la mise en œuvre de l'Accord de Dayton, le Conseil ne maintenait, ni ne menaçait de prendre, aucune sanction à l'encontre de Belgrade eu égard à la situation au Kosovo. Néanmoins, les États-Unis décidèrent unilatéralement de soumettre à un certain nombre de conditions, parmi

\footnotetext{
${ }^{26}$ En mars 1995 , le détachement de l'ONU en Macédoine prit l'appellation de Force de Déploiement Préventif des Nations unies (FORDEPRENU) mais ne devint une opération pleinement indépendante qu'en février 1996, après le démantèlement de la FORPRONU consécutif à la signature de l'accord de Dayton.

${ }^{27}$ Pour l'aider à faire face à l'afflux massif de réfugiés, le gouvernement macédonien put toutefois compter sur les troupes que l'OTAN avait pré-positionnées sur son territoire en prévision d'un accord de paix au Kosovo (KFOR). Tandis que les contigents nordiques de la FORDEPRENU furent rapatriés, le contingent américain vint également renforcer les rangs de la KFOR.
} 
lesquelles la restauration des droits politiques et des droits de l'Homme au Kosovo, l'accession de la RFY à différentes instances internationales, notamment l'ONU, l'OSCE et les institutions de Bretton Woods. Tout en ajoutant quatre exigences préliminaires à la levée dudit " mur extérieur " (outer wall) de sanctions, l'administration Clinton faisait ainsi sienne la volonté d'un certain nombre de parlementaires américains (à commencer par le sénateur républicain Bob Dole) de continuer d'exercer des pressions sur Belgrade concernant la situation dans la province ${ }^{28}$. En 1996, le Congrès précisa dans un nouvel article de loi que le mur extérieur devait demeurer en place jusqu'à ce que le Président des États-Unis l'informe de " progrès significatifs " dans la résolution de la question du Kosovo.

On a fait grand cas de ce dispositif auquel on prêtait des vertus modératrices sur le régime Milošević dont la survie était liée à sa marge de manœuvre financière. Toutefois, le limogeage en mai 1996 de Dragoslav Avramović, le gouverneur de la Banque centrale yougoslave qui préconisait des réformes structurelles au détriment de la nomenklatura de Belgrade, tend à démontrer que le pouvoir en place n'avait aucune intention de se convertir à l'économie de marché et moins encore de se "démocratiser " : il n'allait pas se saborder pour obtenir des crédits du FMI ou de la Banque mondiale. Il est probable en fait que l'administration démocrate prit ces dispositions moins pour faire pression sur Slobodan Milošević que pour répondre aux critiques éventuelles des républicains alors que le Président Clinton se portait candidat à un second mandat. En tout état de cause, les États-Unis n'ont pas cherché à exploiter l'avantage que ces sanctions étaient supposées leur procurer pour obtenir du régime serbe des concessions substantielles. Ils n'ont pas, à titre d'exemple, réclamé le rétablissement au Kosovo d'une présence internationale en charge de surveiller la situation des droits de l'Homme à laquelle ils prétendaient attacher tant d'importance.

En réalité, l'administration américaine est à l'époque surtout soucieuse du sort de ses soldats déployés en Bosnie, dont elle se rend progressivement compte qu'il leur faudra rester sur place plus longtemps que prévu. Elle ne souhaite donc pas s'aliéner le concours de Slobodan Milošević, qui conserve une influence sur les leaders serbes de Bosnie et partant, sur le contexte dans lequel évoluent ses troupes. En outre, pas plus qu'aucun autre le gouvernement amé-

\footnotetext{
${ }^{28} \mathrm{Au}$ total, Belgrade ne pouvait espérer rejoindre les institutions internationales visées qu'à condition de : 1) normaliser ses relations et régler les questions de succession avec les autres républiques issues de l'ex-Yougoslavie, 2) restaurer les droits politiques et les droits de l'Homme au Kosovo, 3) respecter les engagements contractés au titre de l'Accord de Dayton et de sa mise en œuvre, 4) coopérer avec le Tribunal Pénal International pour l'ex-Yougoslavie, 5) entamer un processus de démocratisation en Serbie. Cf. Troebst (Stefan), op.cit., p. 64 ; ICG, Kosovo Spring Report, 24 March 1998 et European Action Council for Peace in the Balkans, the Public International Law \& Policy Group of the Carnegie Endowment for International Peace, Kosovo : From Crisis to a Permanent Solution, Amsterdam/Washington, 1 November 1997, p. 9.
} 
ricain n'est favorable à l'indépendance du Kosovo. Alors que des informations lui parviennent faisant état de l'émergence d'un mouvement albanais de résistance armée, il ne tient pas à prendre des initiatives susceptibles d'encourager ces éléments radicaux. En dépit du Christmas Warning-lancé d'ailleurs en cas d'offensive serbe sur le Kosovo, non pas de provocations albanaises - l'administration Clinton n'avait alors aucune intention d'entamer une nouvelle croisade dans les Balkans. Depuis lors, elle s'était trouvée entraînée en Bosnie, prenait conscience qu'il lui faudrait y demeurer et persistait à croire que s'y trouvait la clef de la stabilité dans la région. En somme, elle n'avait d'autre stratégie pour le Kosovo que d'appliquer l'Accord de Dayton. Au bout du compte, ce mur extérieur n'aidait en rien à résoudre la question du Kosovo (l'amélioration de la situation dans la province ne figurant d'ailleurs qu'une condition parmi d'autres à sa levée) et n'entravait guère la liberté d'action de Slobodan Milošević, mais il confortait l'administration américaine dans l'image de fermeté qu'elle souhaitait donner, à l'adresse en particulier du Congrès. Au demeurant, les Européens passèrent outre à la première occasion venue.

Les Européens n'avaient pas davantage de stratégie pour le Kosovo mais voulaient tout autant croire que la guerre était terminée. Il s'agissait désormais de construire la paix, d'ouvrir un nouveau chapitre et de commencer donc par normaliser les relations avec Belgrade, partenaire obligé de la stabilisation des Balkans. En février 1996 pourtant, à l'image du Congrès américain, le Parlement européen adopta une résolution enjoignant le Conseil de l'Union européenne de conditionner, en tant qu'action commune au titre de la PESC, la reconnaissance de la RFY et la levée des sanctions en suspens à la conclusion d'un règlement satisfaisant entre Slobodan Milošević et les représentants albanais du Kosovo ${ }^{29}$. Dans les deux mois qui suivirent, et en ordre dispersé, la quasi-totalité des États membres reconnut Belgrade. Dès février 1996, la France ouvrit la première le bal des ambassadeurs, suivie - après que la RFY eut normalisé ses relations avec la Macédoine - par la Grande-Bretagne, l'Autriche, l'Allemagne, la Suède, le Danemark, la Norvège, les Pays-Bas, le Portugal, l'Italie et la Finlande. En ouvrant cette brèche dans le mur extérieur de sanctions, les Européens non seulement rompirent la cohésion transatlantique mais affaiblirent un peu plus la position des pacifistes albanais ${ }^{30}$. Au Kosovo, où l'on épiait les faits et gestes du moindre chargé d'affaires occidental, la reconnais-

\footnotetext{
29 Texte reproduit in Troebst (Stefan), op.cit., p. 57.

$3^{\circ} \mathrm{La}$ brèche ouverte dans le mur extérieur de sanctions ne s'entendait pas en termes politiques seulement. Si le régime serbe n'avait nulle intention de se réformer, il avait néanmoins besoin d'élargir sa marge de manœuvre financière afin d'acheter la paix sociale. Il procédait ainsi à des privatisations calculées au plus près et ce sont des entreprises européennes qui venaient alors à son secours - ainsi, en juillet 1996, quand des compagnies grecque, française et italienne lui achetèrent $49 \%$ du capital des postes (bilan de l'opération : cinq milliards de francs versés quelques mois avant les élections; il est vrai que cela ne suffit pas). Cf. Hartmann (Florence), Milošević. La diagonale du fou, Paris : Denoël, 1991, p. 375.
} 
sance de Belgrade confirma en effet les partisans de la lutte armée dans l'idée que, décidément, I. Rugova ne les menait nulle part et qu'il leur fallait sortir de l'impasse, par la force si nécessaire.

\section{La contestation intra-albanaise}

L'Accord de Dayton provoqua une onde de choc au Kosovo, en ce qu'il mettait cruellement en lumière l'échec de la LDK à faire progresser la cause des Albanais sur la scène internationale. À la différence des conférences de La Haye, Londres et Genève au cours desquelles le statut réservé à la délégation albanaise avait déjà suscité des grincements de dents, Dayton ne marquait pas l'ouverture mais bien le terme d'un processus de règlement de la dissolution yougoslave. Aux yeux des Albanais, un accord de paix mettant censément un point final au dossier yougoslave venait donc d'être conclu sans eux - et contre eux. Comment allaient-ils s'en sortir maintenant que les jeux étaient faits ? La colère, la déception, l'angoisse, l'humiliation - tous sentiments qui ne portent guère à la modération - devaient ainsi se répandre au sein de la communauté albanaise et se retourner contre la LDK.

Dayton était un mauvais signal adressé de surcroît à un mauvais moment. Depuis le retrait des Missions de longue durée de l'OSCE, la situation du Kosovo n'avait pas progressé d'un pouce ; celle des Albanais s'était même détériorée à plusieurs égards. En 1992-1993 en effet, la situation générale n'était, en dépit des apparences, pas nécessairement à l'avantage du détenteur de la force. Belgrade n'exerçait qu'un contrôle relatif sur la province, le déploiement massif de policiers et de militaires ne suffisant notamment pas à garantir l'étanchéité des frontières, empêcher les infiltrations d'armes ou assurer la levée des impôts. Contrairement à ses prétentions, le régime s'avérait incapable de venir à bout d'un séparatisme albanais qu'il contribuait au contraire à renforcer. À cet égard, les institutions parallèles mises en place par la LDK étaient un désaveu criant pour les autorités et témoignaient de la vigueur d'une résistance albanaise constituée pour durer. Au fil du temps cependant, le système parallèle commença à montrer ses limites. Si les Albanais parvenaient à subsister grâce à de petits commerces, des activités de service et des trafics en tout genre, 70 \% d'entre eux se trouvaient au chômage et beaucoup survivaient grâce aux allocations de leurs proches travaillant à l'étranger. De fait, la LDK rencontra des difficultés croissantes à recouvrir l'impôt de 3 \% qu'elle percevait de " contribuables " aux revenus réduits et à la bonne volonté déclinante. Il devint ainsi de plus en plus malaisé de maintenir à flot les institutions parallèles mises en place pour pallier les besoins de la population en matière notamment de santé et d'éducation. La détérioration des conditions socio-économiques tendait ainsi à démontrer que le système n'était pas viable et comportait le risque pour la LDK de perdre progressivement le soutien des siens alors que les perspectives politiques ne s'éclaircissaient guère. 
À cet égard, et contrairement à ce que voulurent croire les Occidentaux (et leur faire croire les signataires), l'accord sur l'éducation conclu le $1^{\text {er }}$ septembre 1996 entre Slobodan Milošević et Ibrahim Rugova ne marquait pas une " avancée significative " mais avait été conclu avec moult arrière-pensées des deux côtés ${ }^{31}$. Le Président serbe souhaitait accréditer l'idée qu'il était ouvert au dialogue, mais n'envisageait pas d'accorder la moindre once d'autonomie aux Albanais, dans ce domaine de l'éducation comme en d'autres. Ibrahim Rugova, quant à lui, était animé des mêmes intentions tactiques vis-à-vis des Occidentaux, mais cherchait en sus - comme à l'époque, déjà, des pourparlers de Genève - à améliorer les conditions matérielles d'enseignement des Albanais. En outre, la conclusion d'un accord avec le régime valait à ses yeux reconnaissance par les autorités serbes de sa légitimité et au-delà, de celle de la LDK. En fait, le texte était signé par S. Milošević en qualité de Président de Serbie, mais par I. Rugova en son seul nom, non pas en tant que Président de la " République du Kosovo ". Il l'avait d'ailleurs été séparément par les intéressés, à Belgrade et Priština respectivement, à l'issue d'une longue médiation entreprise pour partie à Rome par la Communauté catholique Sant' Egidio. Au bout du compte, l'accord s'avéra une coquille vide. Il prévoyait le " retour des élèves et professeurs dans les écoles ", mais comme en 1992, ce retour signifiait, pour la délégation serbe, leur réintégration dans le système éducatif de la Serbie alors que les représentants albanais entendaient obtenir la restitution des bâtiments scolaires à leur propre administration. Plus grave encore, les versions détenues par chacune des parties différaient sur un point essentiel, le sort des étudiants, dont le retour était envisagé dans la traduction albanaise, mais non dans l'original rédigé en serbe. Enfin, au titre de la mise en œuvre, le texte prévoyait l'établissement d'une commission mixte de trois représentants par partie mais aucun calendrier. En dépit donc des intentions proclamées par les signataires, l'accord sur l'éducation ne fut pas conclu " au-delà de toutes considérations politiques ", il en était miné. Mais alors que les Occidentaux voulurent y voir un signe, une percée, un espoir, au Kosovo, son échec devait ajouter aux tensions et discréditer un peu plus l'idée même d'un règlement négocié.

Dans l'après-Dayton, Ibrahim Rugova essuya les attaques de plus en plus appuyées de personnalités critiquant son autorité, sa stratégie et jusqu'au cache-misère que constituait à leurs yeux l'État parallèle. Ces contempteurs comprenaient notamment : Bujar Bukoshi, Premier ministre et ministre des affaires étrangères du gouvernement (en exil) de la " République du Kosovo " ; Rexhep Qosja, président du Forum des intellectuels du Kosovo et doctrinaire émérite et sans concessions du nationalisme albanais (prônant ouvertement le 
rattachement de la province à l'Albanie) ; et surtout Adem Demaçi, ledit "Mandela albanais " qui disposait alors, à 60 ans, d'un prestige proportionnel au nombre des années qu'il avait passées dans les geôles de Tito (28) avant d'être libéré en 1990 à la faveur d'une loi d'amnistie. Une opposition hétéroclite mais non moins forte se fit ainsi jour au sommet de la communauté albanaise, qui déplorait que la résistance pacifique fut travestie en résistance passive. Sans se départir encore de la première, cette opposition appelait à substituer à la seconde une résistance enfin active, qui donnerait davantage de corps, d'ampleur et de résultats à la désobéissance civile que le seul boycott des élections et des institutions ${ }^{32}$.

Ces divergences éclatèrent notamment au cours des manifestations de l'opposition serbe de l'hiver 1996-1997. Alors que la LDK avait à nouveau boycotté les élections et considérait leur invalidation comme un problème interne à la Serbie, Demaçi fut le seul dirigeant politique du Kosovo à inviter les Albanais à témoigner de leur solidarité envers les manifestants de Belgrade 33 . A cette occasion, Ibrahim Rugova perdit en sus le soutien sans failles que lui avait accordé jusque-là le président albanais Sali Berisha, lequel se rangea aux côtés de Demaçi. En fait, l'abstention des Albanais jouait si clairement en faveur du pouvoir en place qu'on pouvait suspecter la LDK d'entraver délibérément l'avènement d'une Serbie démocratique afin de préserver la légitimité de la revendication d'indépendance. De fait, Demaçi était aussi le premier à récuser implicitement l'objectif de l'indépendance et prônait plutôt une solution tenant compte des frontières internationales reconnues (une nouvelle confédération baptisée Balkania composée de trois entités souveraines : le Kosovo, la Serbie et le Monténégro). En révisant à la baisse les objectifs à atteindre mais en augmentant l'efficacité de l'action menée, Adem Demaçi entendait ainsi combler le fossé entre les fins et les moyens dans lequel Ibrahim Rugova avait à ses yeux entraîné les Albanais. Cette approche non seulement demeurait pacifique (Demaçi rejetait alors encore la lutte armée), mais la seule à même selon l'intéressé à prévenir la violence à laquelle conduisait tout droit l'attentisme de la LDK.

En octobre 1997, le président de la LDK subit un nouveau désaveu lorsque les étudiants albanais passèrent outre sa recommandation de différer les manifestations qu'ils préparaient dans plusieurs villes du Kosovo afin de réclamer l'accès aux bâtiments universitaires. Descendant dans la rue pour la première fois depuis 1992, les étudiants démontraient ainsi qu'ils ne craignaient plus la police (laquelle intervint toutefois vigoureusement à leur encontre) mais ex-

${ }^{32}$ Rexhep Qosja transgressait toutefois le principe de non-violence en appelant à une résistance sur le modèle de l'intifada. Cf. Troebst (Stefan), op.cit., p. 9.

33 Schmidt (F.), " Protests in Serbia Raise Hopes of Reconciliation in Kosovo ", Transition, 3 (4), 7 March 1997, pp. 16-18. 
primaient aussi leur " ras-le-bol " tant de leurs conditions d'études que de la stratégie stérile à leurs yeux de la LDK. I. Rugova, pour sa part, se trouvait un peu plus coupé de sa base. Le plus grand défi à son autorité était cependant celui lancé par l'UÇK.

\section{L'émergence de I'UÇK}

Sur le terrain, s'affairaient des groupuscules tout aussi déterminés à rompre le statu quo, mais pour lesquels le pacifisme était nécessairement passif et la résistance forcément armée. Dès 1993, des informations confuses avaient fait état de la constitution de forces armées du Kosovo, entraînées dans deux camps du nord de l'Albanie et prévoyant en guise de stratégie de se livrer à des activités de guérilla jusqu'à provoquer l'intervention en leur faveur des Américains ${ }^{34}$. Ce secret espoir de voir les États-Unis leur venir en aide, l'administration américaine l'avait généré en lançant son Christmas Warning à l'adresse de Slobodan Milošević, puis entretenu en intervenant militairement au profit des Musulmans de Bosnie. En février 1996, l'un des groupes incriminés à l'époque par la presse serbe revendiqua les attentats perpétrés dans plusieurs municipalités du Kosovo contre cinq camps de réfugiés serbes 35 . En avril 1996, c'est une mystérieuse " Armée de Libération du Kosovo " (Ushtria Çlirimtare ë Kosoves, UÇK) qui fit parler d'elle en lançant des attaques tout aussi simultanées, c'est-à-dire organisées, à l'encontre de policiers serbes. Pendant encore deux années toutefois, I. Rugova nia l'existence d'une telle organisation clandestine dont il fit porter les méfaits au compte d'une manipulation des services secrets de Belgrade. La montée en puissance de l'UÇK marquait un désaveu cinglant de sa ligne pacifiste, en même temps qu'elle risquait de nuire à la sympathie qu'inspirait à travers le monde la non-violence des Albanais.

En un sens pourtant, et contrairement aux dénégations de I. Rugova, l'Armée de Libération du Kosovo avait toujours existé ou presque. Sous une forme ou sous une autre, sous une appellation ou sous une autre, un mouvement clandestin aspirant à " libérer " le Kosovo, si nécessaire par la violence, s'activait en effet depuis le début des années $1980^{36}$. Au départ, l'Armée de Libération du Kosovo n'était qu'un regroupement ultra-minoritaire d'extré-

34 Cf. Vickers (Miranda), Between Serb and Albanian : A History of Kosovo, London : Hurst \& Company, 1998, pp. 278-279.

$35 \mathrm{Il}$ s'agissait du Mouvement national de libération du Kosovo (Levizja Kombetare per Çlirimin ë Kosoves, LKCK) selon Vickers (Mixanda), op.cit., p. 290. D'autres sources attribuent toutefois cet attentat à l'UÇK.

${ }^{36}$ Sur la genèse de l'UÇK, cf. Chiclet (Christophe), “ Aux origines de l'Armée de libération du Kosovo n, Le Monde diplomatique, mai 1999 et " UÇK : les vrais maitres du Kosovo ", Politique internationale, (85), automne 1999 ; Allain (Marie-Françoise), "L'UÇK : de l'ombre au grand jour n, in Garapon (Antoine), Mongin (Olivier), éds., Kosovo, un drame annoncé, Paris : Michalon, 1999, pp. 227-243 ; ainsi que Judah (Tim), Kosovo. War and Revenge, New Haven/London : Yale University Press, 2000, pp. 102-120. La chronologie donnée par ces trois auteurs diffère parfois sensiblement. 
mistes pénétrés de marxisme-léninisme et de nationalisme pan-albanais, par ailleurs soupçonnés de verser dans la criminalité organisée entre la Suisse, l'Allemagne et le Kosovo. L'UÇK devait toutefois parvenir à s'implanter dans les régions rurales de la province, en particulier dans ce bastion traditionnel de résistance à l'ordre étatique (turc d'abord, serbe ensuite) qu'est la vallée de Drenica. Alors que la LDK disposait du soutien des intellectuels et des milieux urbains, l'UÇK recrutait dans les campagnes où le sens de l'honneur, du sang versé et du secret lui assurait complices et complicités. En 1996-1997, son existence même faisait cependant encore l'objet de spéculations et son effectif n'était estimé qu'à quelques centaines de combattants. Son essor, l'UÇK le dut, outre l'oppression continue du régime serbe et l'échec de la résistance pacifique, à l'effondrement de l'Albanie.

Le naufrage dans lequel sombra l'Albanie au printemps 1997 sonna, provisoirement du moins, le glas des aspirations à l'unification des Albanais disséminés dans les Balkans. Mais en vérité, avant même que l'État albanais ne se dévoile dans toute sa vacuité, il n'avait jamais constitué un point d'appui pour les Albanais de l'extérieur, ni même pour ses propres citoyens (auxquels il inspire plutôt la tentation de le fuir) ${ }^{37}$. Cet attachement pour le moins mesuré à leur État-patrie tend à relativiser les aspirations pan-nationales dont les Albanais, de l'extérieur en particulier, sont soupçonnés. Du reste, la Grande Albanie tient d'autant plus du mythe que la " petite " n'a jamais eu les moyens financiers ni militaires (et moins encore après mars 1997) de venir en aide aux populations albanaises établies hors de ses frontières. Toutefois l'effondrement de l'Albanie devait, paradoxalement, précipiter l'éclatement du conflit du Kosovo. À cette occasion en effet, le pillage des arsenaux albanais par les émeutiers se traduisit par la disparition de plus de 600 ooo armes de tous calibres et de 800 millions de dollars de matériel militaire $3^{8}$. Nombre de ces armes tombèrent entre les mains des militants de l'UÇK, pour lesquels le nord de l'Albanie, échappant désormais au contrôle de Tirana, servit par ailleurs de base arrière. Si l'opération Alba déployée sous commandement italien contribua à ramener un semblant de normalité dans le pays, les États participant à la force renoncèrent à récupérer les armes en circulation (une tâche il est vrai titanesque). Par la suite, aucune proposition ne fut non plus présentée au gouvernement pour contrôler les frontières septentrionales de l'Albanie, bien que Tirana eût officiellement demandé l'établissement d'une présence de l'OTAN

\footnotetext{
${ }^{37}$ Les Albanais de l'extérieur vivent une " territorialité èclatée autour d'un centre vide n expliquent ainsi Deslondes (Olivier), Rapper (Gilles de), Roux (Michel), " Les Albanais hors d'Albanie. Émigrés et minorités en Grèce, en Macédoine et en Yougoslavie n, Hérodote, 98 (3), 1998, pp. 29-30.

${ }^{38}$ Chiffres du ministère albanais de la défense cités in Albania Human Development Report 1998, UNDP, p. 73. Certains analystes estimèrent même qu'entre 750 ooo et un million d'armes légères disparurent. Cf. Smith (Chris), Sagramoso (Domitilla), " Small arms trafficking may export Albania's anarchy n, Jane's Intelligence Review, 11 (1), January 1999, p. 25.
} 
dans cette zone. Ainsi, l'un des objectifs de l'offensive lancée par Belgrade au cours de l'été 1998 sera précisément de " sécuriser " cette frontière, afin d'empêcher l'UÇK de s'approvisionner à Kukës et Tropojë.

\section{À LA RECHERCHE D'UNE STRATÉGIE PRÉVENTIVE POUR LE KOSOVO}

Comme pour donner raison à l'UÇK que la " communauté internationale " n'entend que le langage de la force, un certain activisme diplomatique se développa au cours de l'année 1997 à mesure que parvinrent des signes de détérioration en provenance du Kosovo et se précisait en particulier l'émergence d'un mouvement albanais de résistance armée. Anticipant sur cette évolution, nombre d'experts et d'observateurs avaient pris bien plus tôt la mesure du caractère explosif de la situation au Kosovo et notamment des risques d'aggravation engendrés par la signature de l'Accord de Dayton. Différentes initiatives furent ainsi lancées dès 1996 dans les milieux académiques et non gouvernementaux pour tenter de définir une stratégie de prévention. C'est à l'aune des recommandations formulées par quelques-uns de ces groupes de réflexion que l'on se propose ici de mesurer les tentatives ultérieures d'intervention des acteurs gouvernementaux 39 .

\section{Conseils d'experts}

De l'avis général des experts et ONG, aucune " solution " n'était envisageable à court terme susceptible de mettre un point final à la question du Kosovo. En théorie certes, le statut accordé à la province, qui constituait (et constitue toujours) le nœud du problème, pouvait revêtir de multiples formes mais aucune des options avancées ne rencontrait l'adhésion conjointe des autorités serbes et des représentants albanais. Des deux côtés, des solutions médianes en apparence furent évoquées qui conciliaient le respect de l'intégrité territoriale de la RFY avec une certaine latitude laissée au Kosovo. Mais il demeurait un gouffre entre le statut d'État souverain promu par A. Demaçi au sein d'une nouvelle confédération "balkanique " et l'entité plus ou moins décentralisée mais dépourvue de personnalité étatique que les partis d'opposi-

39 On se réfère ici aux travaux suivants : Aspen Institute Berlin, Carnegie Endowment for International Peace, Unfinished Peace, Report of the International Commission on the Balkans, 1996, pp. 112-119; Rubin (Barnett R.), ed., Towards Comprehensive Peace in the Southeast Europe : Conflict Prevention in the Balkans (Report of the South Balkans Working Group of the Council on Foreign Relations Center for Preventive Action), New York : Twentieth Century Fund Press, 1996 ; European Action Council for Peace in the Balkans, the Public International Law \& Policy Group of the Carnegie Endowment for International Peace, op.cit. ; Bertelsmann Science Foundation \& Research Group on European Affairs, Joint Recommendations on the Kosovo Conflict, 10 December 1997. Les conclusions de ces travaux (et d'autres initiatives non-gouvernementales) sont reproduites dans le recueil de Troebst (Stefan), op.cit. 
tion serbe se disaient prêts à accepter. Au demeurant, ces options intermédiaires étaient rejetées par les forces majoritaires de chacun des deux camps : par la LDK, qui ne démordait pas de l'objectif d'indépendance et par la coalition au pouvoir à Belgrade, qui réprouvait toute modification de la Constitution serbe et entendait plutôt renforcer les compétences des institutions fédérales. Suggérée par l'Académie serbe des sciences et des arts en juin 1996, la partition du Kosovo fut rejetée comme une trahison dans les cercles nationalistes serbes, comme un abandon par les Serbes du Kosovo et fut réprouvée par les Albanais qui certes, apprécièrent l'idée comme une marque de défaitisme, mais entendaient préserver, pour leur compte, l'intégrité territoriale de la province. En fait, Serbes et Albanais de toutes tendances ne s'entendaient que sur un point : le rejet du statut d'autonomie de 1974 et de toute formule tendant à s'en rapprocher - les premiers, parce que l'expérience avait à leurs yeux nourri le séparatisme des Albanais et la discrimination des Serbes ; les seconds, parce qu'elle s'était soldée par la révocation dudit statut et l'imposition à leur encontre d'un régime d'apartheid.

Dans ces conditions, toute tentative de médiation axée sur la recherche d'une solution au problème du statut était vouée à l'échec. S'imposait et se vérifiait au contraire dans le cas du Kosovo une approche de la prévention conçue moins comme une procédure de règlement définitif qu'un processus de changement pacifique. Si ce processus visait in fine à formuler une solution mutuellement acceptable et donc durable, celle-ci n'en était pas l'objectif immédiat ni surtout, ne devait être définie ou seulement esquissée par avance. Il incombait aux parties de s'entendre à terme sur les modalités d'un règlement et non pas au médiateur international - quel qu'il fut - d'en fixer dès le départ les grandes lignes. Dans l'intervalle, les efforts de prévention devaient se concentrer d'une part, sur l'amélioration du contexte général et d'autre part, sur l'ouverture d'un dialogue entre les protagonistes. Dans cette perspective, les autorités serbes et les représentants albanais devaient être incités à prendre des initiatives qui marqueraient le début du processus. Du côté de Belgrade, il s'agissait de lever la loi martiale imposée au Kosovo et autres décrets pris au titre des " circonstances spéciales ", de procéder au retrait progressif des forces de police et de l'armée, et enfin de mettre un terme aux actes de répression et violations des droits de l'Homme. C'était sans doute beaucoup demander aux autorités, mais elles seules faisaient alors usage de la force et c'était un minimum pour entamer des pourparlers sur des bases saines. Les représentants albanais de leur côté devaient réaffirmer leur attachement à la non-violence, renoncer à faire de l'indépendance le seul sujet et la seule issue acceptables des discussions, enfin certifier de leur bonne disposition vis-à-vis de la population serbe du Kosovo. Afin de vérifier la suite donnée à ces engagements et contribuer à détendre l'atmosphère, était requise une présence internationale sur le terrain (de l'ONU, l'OSCE ou l'Union euro- 
péenne) dont l'acceptation dépendait évidemment de Belgrade et s'inscrivait dans le cadre des " mesures de confiance " que devait prendre le régime. Les deux directions (contexte et dialogue) convergeaient alors au sens où des mesures concrètes pouvaient être négociées par les parties, qui constitueraient autant de sujets de discussions tout en améliorant les conditions économiques et sociales prévalant dans la province. Au-delà même, ces dispositions pouvaient s'inscrire dans un règlement provisoire et leur application relever d'une autorité intérimaire conjointe. Ce n'est qu'après ce long parcours, l'apaisement subséquent des tensions, l'amélioration sensible du contexte et le changement éventuel des dirigeants, que pouvait commencer d'être négociée une formule de compromis sur le statut de la province. Au moins, ce processus devait-il permettre de se rapprocher d'un règlement et se trouvait théoriquement écarté pendant sa durée le risque que la situation ne dégénère en conflit armé.

Se posait la question de savoir à qui incomberait le rôle de tierce partie. Dans tous les cas, discrétion et confidentialité s'imposaient, au début au moins du processus. À cet égard, l'idée lancée par certains de convoquer une conférence internationale de type " Dayton II " était totalement inadaptée, en ce qu'elle ajoutait au caractère prématuré de l'exercice les inconvénients de la surexposition médiatique. La diplomatie dite de " seconde voie " (track two diplomacy) se prêtait davantage aux circonstances, mais l'expérience de Sant' Egidio en avait montré les limites et l'on ne pouvait guère escompter d'une organisation non gouvernementale qu'elle parvienne, en son seul nom, à convaincre le régime serbe de retirer ses forces du Kosovo. À la confidentialité devait nécessairement s'ajouter un solide pouvoir de persuasion, dont seuls disposent les acteurs gouvernementaux. Ne restait donc que deux options : l'intervention d'un médiateur fort du soutien d'un groupe d'États mais agissant en coulisses, ou bien celle d'un groupe d'États, qui tente d'exercer des pressions et détache, le cas échéant, un médiateur. Au cours de l'année 1997, les deux principales initiatives prises à l'échelle internationale pour tenter d'enrayer la crise qui se développait au Kosovo incombèrent ainsi au Haut Commissaire de l'OSCE pour les minorités nationales puis, à partir de septembre, aux six États composant le Groupe de Contact. La première illustre tout ce que la diplomatie préventive ne peut pas faire et la seconde tout ce qu'il ne faut pas faire en matière de diplomatie préventive.

\section{Les mésaventures de Max van der Stoel}

Le poste de Haut Commissaire pour les minorités nationales (HCMN) fut créé à l'issue de la Conférence d'examen d'Helsinki de 1992 avec pour fonction de déclencher une " alerte rapide " et, si besoin, d'engager une " action rapide " lorsque surgissent des tensions liées à des problèmes de minorités nationales susceptibles, selon le HCMN, de dégénérer en conflit ouvert dans la zone de 
l'OSCE $^{\circ}$. Le premier titulaire du poste, le diplomate néerlandais Max van der Stoel, prit ses fonctions en janvier 1993. Alors toutefois que la situation au Kosovo était typiquement passible de son intervention, l'intéressé s'abstint de toute initiative à ce sujet jusqu'à ce que la Présidence de l'OSCE le charge, en février 1997, d'étudier les moyens de réduire les tensions et d'explorer les possibilités de dialogue entre les autorités de Belgrade et les représentants des Albanais. Du reste, $M$. van der Stoel devait agir en qualité de Représentant personnel pour le Kosovo du Président en exercice de l'époque, le Danois Niels Helveg-Petersen, non pas en tant que Haut Commissaire pour les minorités nationales. En règle générale toutefois, le $\mathrm{HCMN}$ ne s'investissait que dans les situations à l'amélioration desquelles il estimait pouvoir contribuer, après avoir discrètement sondé la disposition des parties à son égard. S'il ne se saisit pas de sa propre initiative de la question du Kosovo, c'est donc probablement qu'à ses yeux, les circonstances ne s'y prêtaient pas. La suite devait rapidement lui donner raison. Non seulement les protagonistes étaient parfaitement réfractaires les uns aux autres, mais ils manifestèrent une commune hostilité à l'intervention annoncée de $M$. van der Stoel.

Le régime de Belgrade venait alors de reconnaître sa défaite aux élections municipales, qu'un autre émissaire de l'OSCE justement (Felipe Gonzales) avait attestée à ses dépens. Il n'avait pas l'intention d'accepter une nouvelle ingérence de cette organisation et moins encore (excluant depuis quatre ans d'y rétablir les MLD) à propos du Kosovo. Le Représentant spécial se vit ainsi refuser un visa jusqu'en février 1998, à savoir un an après sa nomination et quelques jours avant l'offensive serbe dans la vallée de la Drenica marquant le début de la guerre. Les autorités de RFY, qui accédaient ce faisant à une requête du Conseil ministériel de l'OSCE, avaient sans doute alors dans l'idée de renouer avec leur tactique habituelle, consistant à souffler le chaud et le froid et générer des illusions sur leur volonté de négocier. Mais en vérité, la visite que le HCMN fut autorisé à effectuer ne s'entendait qu'à titre " privé " et ne lui permit de rencontrer, à Belgrade, qu'un haut fonctionnaire du ministère fédéral des affaires étrangères.

Les représentants albanais étaient également très réservés à l'égard de $M$. van der Stoel, qu'ils percevaient avant tout comme le Haut Commissaire pour les " minorités ". Or, il était de notoriété publique, depuis au moins la création du Groupe de travail de la CIEX, qu'aborder la question du Kosovo en ces termes était le meilleur moyen de s'aliéner leur coopération. Les Albanais constituaient en effet la majorité numérique de la province et considéraient irréductible à un problème de minorité leur situation de peuple asservi par une puis- 
sance occupante. Adem Demaçi refusa ainsi de rencontrer le HCMN. Bref, les " possibilités de dialogue " apparaissaient bien maigres.

En octobre 1997, le Représentant personnel parvint certes à organiser en Autriche des consultations entre personnalités serbes de Belgrade et albanaises de Priština, mais s'il incluait des membres de la LDK (et notamment son vice-président Fehmi Agani), ce forum ne comptait aucun dirigeant politique serbe et ne pouvait servir de substitut à d'authentiques pourparlers entre représentants légitimes ${ }^{41}$.

Après le déclenchement des hostilités en mars 1998, M. van der Stoel fut dessaisi du dossier au profit de Felipe Gonzales, nommé pour sa part Représentant personnel du Président en exercice pour la République fédérale de Yougoslavie (il ne mit pas davantage les pieds en RFY et finit par se désigner lui-même " l'Envoyé invisible "). En final, les efforts entrepris par le diplomate néerlandais s'étaient soldés par un échec complet. Outre que la nomination d'un médiateur ne suffisait en elle-même à débloquer la situation, celle de $M$. van der Stoel apparait après coup peu judicieuse. Ce n'est certes pas la personne qui est en cause, mais plutôt le rôle qu'on lui fit jouer, la double casquette qu'on lui fit porter. Le HCMN fut sollicité par le Président danois en raison de ses talents de négociateur, de son impartialité et de son expertise en matière de minorités. Mais le nommer Représentant personnel, c'était le priver de son autonomie traditionnelle à l'égard des États participants et conférer à son action un caractère par trop formel, c'était lui ôter ce qui faisait d'ordinaire sa force : l'indépendance et la confidentialité. Représentant en outre une organisation dont la RFY demeurait suspendue, $M$. van der Stoel se retrouvait en porte-à-faux avec les autorités de Belgrade. A l'inverse, le Représentant personnel restait aux yeux des Albanais le HCMN. Paradoxalement donc, l'expérience de $M$. van der Stoel en matière de minorités se retourna contre lui, au point que sa nomination s'apparente à une erreur politique. Ajoutons qu'au cours des douze mois que dura cette mission, le climat général ne cessa de se dégrader dans la province en conséquence des attentats commis par l'UCK et de la réaction musclée du régime serbe aux manifestations, pacifiques cellesci, des étudiants albanais.

\section{L'approche biaisée du Groupe de contact}

C'est précisément en réponse à ces développements que le Groupe de contact émit le 24 septembre 1997 sa toute première déclaration sur le Kosovo

${ }^{41}$ Ce forum réunissait en fait les participants « habituels n, réputés pour leur intellect et leur ouverture d'esprit, des rencontres sur le Kosovo organisées par des ONG ou des groupes de travail - ainsi côté albanais de Shkëlzen Maliqi (directeur de l'Open Society Institute à Priština), Veton Surroi (rédacteur en chef de Koha Ditore) ou Gazmend Pula (directeur du comité Helsinki pour les droits de l'Homme au Kosovo) et côté serbe de l'universitaire Predrag Simić. 
de l'après-Dayton ${ }^{42}$. Ce texte est d'importance car le Groupe de contact ne figurait pas seulement un forum informel de concertation, mais devait s'imposer en raison de sa composition comme une véritable instance de décision, une sorte de Conseil de sécurité pour les Balkans dont les prises de position détermineront en grande partie l'action multilatérale dans la région. Il servira ainsi d'antichambre au Conseil de sécurité des Nations unies (dont il rassemblait quatre des cinq membres permanents), mais aussi au Conseil permanent de l'OSCE (dont les autres États participants suivraient ses orientations).

Or, cette première déclaration, déjà bien tardive en soi, augurait mal d'une stratégie préventive efficace pour le Kosovo. Alors que les " experts " recommandaient de s'attacher à enclencher un processus, mais de s'abstenir d'en définir le résultat, le Groupe de contact adopta la démarche exactement inverse. Il se prononça en effet avec clarté sur le fond en récusant tant l'indépendance que le statu quo et prônant un statut " amélioré " au sein de la RFY. En apparence, ce semblait être une proposition médiane, équilibrée et pour tout dire impartiale. En vérité, le Groupe de contact prenait lui-même parti en exprimant ses préférences, il prônait une solution irréaliste en la circonstance puisque rejetée par l'ensemble des protagonistes et ne prenait par ailleurs pas la moindre initiative pour tenter de débloquer la situation. Plutôt, en effet, que de chercher à réduire les tensions pour qu'un dialogue puisse à terme s'instaurer, le Groupe de contact appelait au dialogue afin de réduire les tensions. C'était un vœu pieux que n'appuyait nulle mesure incitative ni pression d'aucune sorte. Sa politique se résumait à renvoyer dos à dos le régime serbe et les Albanais (sans distinction aucune) ainsi qu'à promouvoir une option confirmant le premier dans l'idée qu'il se faisait de ses droits sur le Kosovo sans entamer en rien la détermination des seconds à conquérir les leurs. En fait, le Groupe de contact renouait avec la même impartialité biaisée qui avait innervé la politique occidentale au cours du conflit de Bosnie. La philosophie générale de cette déclaration était celle d'une équité parfaite entre les autorités serbes et les représentants des Albanais, qui tranchait singulièrement avec l'asymétrie des rapports de force. Exhortant "l'ensemble des parties à faire preuve du maximum de retenue ", le Groupe de contact ne portait nullement crédit à la LDK de ce se conformer à une ligne pacifique - ce qui confirmait l'UÇK dans le bien-fondé de la lutte armée - et n'appelait que de façon oblique les autorités de Belgrade à tempérer leur réaction. Peu après, l'usage, pour la première fois, du terme " terroriste " pour qualifier les actions de l'UÇK était lui dépourvu d'ambiguïté43. Le Groupe de contact tranchait ainsi ce dilemme de

$4^{2}$ " Statement on Kosovo of the Contact Group Foreign Ministers n, OHR Document, 24 September 1997. Formé en 1994 pendant la guerre de Bosnie, le Groupe de contact était composé des États-Unis, de la Russie, de la France, de la Grande-Bretagne, de l'Allemagne et, après la crise albanaise, de l'Italie.

43 Cf. " Contact Group Meeting, Statement on Kosovo ", OHR Document, 8 January 1998. 
savoir comment considérer cette armée de l'ombre, comme un groupe terroriste ou un mouvement de libération nationale44. Il avait sans doute des raisons d'opter pour la première option, mais en reprenant à son compte le vocabulaire de Belgrade, il s'alignait sur la position du régime serbe et tendait à légitimer, sinon encourager, la stratégie " anti-terroriste " des autorités. On était loin alors du Christmas Warning lancé en son temps par l'administration américaine (et tout autant en fait de l'opération " Force Alliée ").

Le 8 janvier 1998, le Kosovo fut érigé au rang de " priorité " par les six États concernés. Dans la foulée, fut mis en place un "groupe de travail spécifique " qui se réunit pour la première fois le 5 février suivant. Autrement dit, ce n'est que quatre ans après sa création, deux ans après la signature de l'Accord de Dayton, et quelques semaines avant l'éclatement des hostilités dans la province, que le Groupe de contact convint d'établir une structure permanente dédiée au problème du Kosovo. En définitive, la règle du "trop peu, trop tard " s'est à nouveau imposée. Pourquoi en fut-il ainsi ? On peut avancer différents éléments d'explications.

En premier lieu, le Groupe de contact prenait certes conscience de la montée des tensions dans la province mais pas nécessairement de l'imminence d'un conflit armé. Peut-être pensait-il ainsi disposer d'un certain délai, auquel cas il se trompait lourdement. En second lieu, l'idée même de concilier prévention et coercition se heurtait à de sérieuses résistances, tant des Occidentaux pour lesquels une stratégie coercitive ne s'entend qu'en dernier ressort, que des Russes ou des Chinois qui bornent l'action préventive - s'agissant $a$ fortiori d'une situation interne - à la seule diplomatie. À l'évidence, ces conceptions limitatives et divergentes entravaient tout accord au sein du Groupe de contact, et au-delà du Conseil de sécurité, sur l'adoption d'une stratégie un tant soit peu musclée. En troisième lieu, les Occidentaux étaient alors très réticents à l'idée d'entamer une épreuve de force avec Slobodan Milošević. Celui-ci leur apparaissait plus que jamais incontournable étant donné l'étendue de ses pouvoirs, la vacuité de l'opposition et surtout, la présence en embuscade d'un Vojislav Šešelj - le leader radical avait échoué de peu à se faire élire à la présidence de Serbie - avec lequel ils concevaient mal d'obtenir un accord sur le Kosovo. Toute solution passant par S. Milošević, il ne s'agissait pas de s'aliéner son concours en adoptant un profil par trop offensif et moins encore de risquer de provoquer sa chute. Ce concours, les Occidentaux en ressentaient de surcroît le besoin pour préserver l'application de l'accord de Dayton. Le 22 février 1998, l'admi-

$44 \mathrm{Il}$ ne se posa sans doute pas longtemps la question car l'ensemble de ses membres considéraient les Albanais du Kosovo comme une minorité. Or, en droit international, seuls les " peuples " peuvent être représentés par un mouvement de libération nationale. Cf. Kohen (Marcelo), "L'emploi de la force et la crise du Kosovo : vers un nouveau désordre juridique international ", in Morand (Charles-Albert), éd., La crise des Balkans de 1999. Les dimensions historiques, politiques et juridiques du conflit du Kosovo, Bruxelles : Bruylant [coll. Axes n²2], 2000, pp. 138-139. 
nistration américaine jugea ainsi opportun de récompenser Belgrade pour le soutien que faisait mine d'apporter Slobodan Milošević au nouveau Premier ministre modéré de Republika Srpska, Milorad Dodik, en levant quatre interdictions frappant la RFY45. Pour beaucoup, Slobodan Milošević interpréta ces concessions comme un feu vert à l'offensive qu'il s'apprêtait à lancer dans la province contre les " terroristes " de l'UÇK - dont les agissements étaient tout aussi vigoureusement dénoncés par le Groupe de contact. En dernier lieu, la formulation d'une stratégie coercitive se heurtait à un obstacle de taille : fondamentalement, Américains, Russes et Européens partageait l'opposition de Belgrade à l'indépendance du Kosovo et souhaitaient tout autant que les combattants de l'UÇK fussent mis hors d'état de nuire. En définitive, les Occidentaux (et moins encore les Russes) n'avaient nullement l'intention de menacer de la force ou toute autre sanction un régime qu'ils croyaient pouvoir influencer, avec lequel ils souhaitaient préserver de bons rapports et dont ils partageaient les objectifs stratégiques. Leur but était de parvenir à une solution au plus vite, en poussant les représentants albanais à composer, comme eux, avec Belgrade. Mais de solution, il n'y en avait pas et l'approche purement coopérative laissait libre d'agir à sa guise un régime essentiellement belliciste. En la circonstance, si les Occidentaux voulaient la paix, il leur fallait préparer la guerre. À défaut, et contrairement à leurs intentions initiales, ils durent la faire.

\section{Aurait-il pu en être autrement ?}

"C'est facile avec le recul. " Telle est sans doute la réflexion qu'inspire ce compte-rendu critique de la diplomatie préventive au Kosovo. Effectivement, c'est le privilège de l'historien que de bénéficier de la vision rétrospective et d'ainsi reconstituer, de façon parfois artificielle parce que trop linéaire, le fil de l'Histoire. Mais c'est la responsabilité du politique que d'anticiper, de voir large et de voir loin, et d'agir en conséquence. Or, alors que Dayton s'apparentait à bien des égards à une invitation à la guerre, que de nombreux observateurs convertirent l'invitation en avertissement et relevèrent, dès 1996, qu'un bien mauvais signal avait été adressé aux " radicaux " et un bien mauvais service rendu aux " modérés ", les gouvernements occidentaux ne réagirent qu'avec un retard consommé à la montée des périls dans la province et prirent ensuite le contre-pied d'à peu près chacune des recommandations formulées par le milieu non gouvernemental pour enrayer la crise.

45 Jusqu'alors la RFY ne pouvait participer à l'initiative de coopération en Europe du Sud-Est (SECI), ouvrir un consulat aux États-Unis, ni recruter du personnel pour sa représentation permanente à l'ONU et les appareils de la compagnie aérienne yougoslave (JAT) n'avaient pas l'autorisation d'atterrir sur des aéroports américains. 
Au demeurant, ces recommandations étaient peut-être quelque peu tronquées en ce que la prévention d'un conflit au Kosovo ne se heurtait pas à un problème de confiance ou de méthode mais butait sur un déficit flagrant de volonté politique des deux côtés. Coté albanais, la LDK demeurait certes attachée à la non-violence mais n'envisageait nullement d'acheter la paix au prix de l'indépendance. Elle s'accrochait d'autant plus à cet objectif que tout reniement de sa part aurait achevé de la discréditer. Côté serbe, si le régime avait pu un temps se contenter par défaut du statu quo, il n'avait jamais manifesté l'intention de régler la question du Kosovo de façon négociée et moins encore à mesure qu'il accumulait les déboires électoraux. Dès lors, il était illusoire d'escompter de Slobodan Milošević qu'il consente à lever l'état de siège, accepte une présence internationale dans la province ou se conforme à d'autres demandes encore (comme restaurer le statut d'autonomie) auxquelles il s'était farouchement opposé depuis des années. Dans ces conditions, et les mésaventures de $M$. van der Stoel en témoignent, tout processus coopératif de prévention fondé sur " les intérêts bien compris " des intéressés était voué à l'échec. $\mathrm{Au}$ demeurant, le groupe de travail de la CIEX en avait déjà fait l'expérience, avant même que l'UÇK ne vienne ajouter aux difficultés. L'idée en particulier que des pourparlers puissent s'engager sans préjudice des positions politiques s'était avérée chimérique.

Ceci n'est pas dire que le processus préconisé par les think tanks ne faisait pas sens. Au contraire, il y avait lieu d'obtenir au préalable à toute discussion la levée de l'état de siège ainsi que d'ajourner la définition du statut final du Kosovo. Mais ledit processus ne pouvait voir le jour qu'à condition de contraindre et non seulement d'inciter les parties récalcitrantes à y participer. En d'autres termes, la situation obligeait à formuler une stratégie coercitive, visant non pas à imposer un règlement mais à empêcher d'agir les partisans du recours à la force et ce faisant, à pousser autant qu'il était possible l'ensemble des protagonistes à s'engager sur la seule voie qui leur resterait : la voie diplomatique. La tâche, on en convient volontiers, n'était pas aisée, ni n'offrait aucune garantie de succès. Mais elle correspondait sans doute davantage aux exigences de la situation que les dérisoires appels au dialogue lancés par le Groupe de contact. Dans cette perspective, celui-ci aurait par exemple pu réitérer l'avertissement lancé en 1992-1993 à l'adresse du régime serbe. Il aurait pu donner corps à cette menace, soit en effectuant des démonstrations de force (aérienne), soit en procédant au détachement de troupes (de vraies troupes) en Macédoine et en Albanie. Ce double déploiement préventif aurait assumé une fonction dissuasive à l'égard de Belgrade en préfigurant une éventuelle intervention terrestre. Les troupes engagées auraient également pu fournir une assistance aux deux pays-hôtes en cas d'éclatement des hostilités. En Macédoine, le renforcement de l'effectif et du mandat de la FORDEPRENU aurait pu suffire, en même temps qu'il aurait enfin donné un sens à la mission des " casques 
bleus " sur place. En Albanie, la force déployée aurait aidé, comme il l'avait demandé, le gouvernement à rétablir son autorité sur le nord du pays, à reprendre le contrôle des frontières et lutter contre le trafic d'armes ${ }^{46}$. Cette stratégie coercitive aurait en effet eu comme objectif corrélatif d'empêcher l'UÇK de nuire, notamment en la privant de ses bases arrières et sources d'approvisionnement en Albanie. Sur le front politique, le Groupe de contact aurait pu justifier ses décisions en dénonçant tant les activités terroristes de l'armée de libération que le régime de terreur imposé par Belgrade aux Albanais. Et plutôt que promouvoir une solution irréaliste à la question du statut, il aurait pu commencer par condamner les violations des droits de l'Homme perpétrées dans la province puis exiger, sous peine de sanctions économiques et financières ciblées, qu'un terme immédiat y soit mis et que l'état d'urgence soit levé. Il aurait pu, enfin, exprimer l'idée que l'exercice de la souveraineté étatique impose à son dépositaire, comme à tout aspirant, l'obligation irréfragable de respecter les droits de l'Homme et les libertés fondamentales. Toutes ces décisions et prises de position auraient été, " naturellement ", avalisées par le Conseil de sécurité, constatant en vertu du chapitre VII de la Charte des Nations unies l'existence au Kosovo d'une menace à la paix et la sécurité internationales.

$\mathrm{Ce}$ ne sont là que quelques pistes qu'il est vain d'explorer plus avant. Au demeurant, on convient volontiers qu'une telle approche n'aurait pas nécessairement suffi à faire plier le régime serbe puisqu'en dernière instance, celuici n'accepta une présence internationale au Kosovo qu'après soixante-dix-huit jours de bombardements. Pour qu'une stratégie de nature coercitive se mette en place, il aurait en outre fallu que les acteurs concernés (en substance, les pays membres du Groupe de contact) surmontent un certain nombre des obstacles qui entravèrent leur action : qu'ils adoptent une position moins dogmatique sur le statut du Kosovo et se fixent pour objectif premier d'éviter un conflit dans la province plutôt que s'arc-bouter sur la question de l'indépendance ; qu'ils cessent de considérer Slobodan Milošević comme un acteur incontournable ou voient aussi en lui le criminel de guerre qu'il était dès avant le printemps 1999 ; qu'ils coordonnent leurs politiques plutôt que de privilégier leurs intérêts nationaux ; qu'ils ne redoutent pas de prendre des risques en déployant des troupes en Albanie, ni ne craignent d'exposer celles déjà présentes en Bosnie ; qu'ils s'entendent, Russie comprise, sur des mesures de contrainte à exercer contre Belgrade et obtiennent, au Conseil de sécurité, le ralliement de la Chine. Bref, il aurait fallu que se manifeste une volonté collective. Or, c'est bien davantage l'improvisation qui caractérisa la diplomatie internationale dans les Balkans au cours de la décennie 1990. Contrairement, en effet, aux théories du complot qui firent florès lors de l'opération " Force

46 À cet égard l'Assemblèe parlementaire de l'UEO avait adopté en novembre 1997 une recommandation invitant le Conseil de l'organisation à planifier le déploiement d'une force européenne en Albanie. 
Alliée ", les gouvernements occidentaux pêchèrent par manque d'anticipation (non par vanité stratégique) et furent en final bien davantage entraînés par les événements qu'ils ne parvinrent à en infléchir le cours. Ainsi, outre qu'ils se compromirent auprès d'une guérilla dont ils récusaient initialement tant les objectifs que les méthodes, les États-Unis furent amenés à déployer de nouvelles troupes dans les Balkans ; ils ne le voulaient pas. Les Européens, et notamment les Français, durent laisser l'OTAN recourir à la force sans mandat explicite des Nations unies ; ils ne le voulaient pas davantage. Les Russes assistèrent à la première intervention " hors-zone " de l'Alliance atlantique contre un État souverain et subirent à cette occasion une humiliation publique; ils ne voulaient pas non plus. Si la puissance d'un État est bien sa capacité à imposer sa volonté, alors les dites " grandes puissances " ont, au cours de la décennie, fait principalement étalage de leur impuissance - et de leurs divisions.

\section{CONCLUSION GÉNÉRALE}

Les initiatives préventives prises en RFY et en Macédoine s'inscrivaient initialement dans une perspective régionale. Il s'agissait d'éviter qu'à la suite d'une agression serbe ou de l'explosion d'un conflit au Kosovo, la Macédoine ne soit à son tour plongée dans le chaos et n'entraîne dans sa suite ses quatre voisins. Le scénario du pire ne s'est pas produit, mais un scénario catastrophe s'y est substitué : la guerre du Kosovo a bien eu lieu et en fait de conflit régional entre quatre États se déchirant la Macédoine, dix-neuf États sont entrés en guerre contre la Serbie - sans pour autant empêcher la première de finalement se déchirer. L'histoire est d'autant plus cruelle que ces dix-neuf, ou certains d'entre eux, s'étaient fixé pour objectif d'empêcher les quatre premiers de recourir à la force. Quoi qu'on pense de la légitimité de l'intervention de l'OTAN, le bilan en termes de prévention n'est pas brillant. À l'évidence, le conflit du Kosovo constitua un échec indéniable de la diplomatie préventive, retentissant même au regard des proportions qu'il prit. Cet échec s'explique par des facteurs à la fois endogènes et exogènes : d'une part, il faut reconnaître que ce conflit était des plus difficiles à prévenir ; d'autre part, la politique préventive qui fut menée s'avéra largement inadaptée à la situation.

À bien des égards, le Kosovo constituait un terrain des plus défavorables à l'action préventive, un cas extrême, presque caricatural et fort singulier : celui d'une minorité constituant à tous égards une population à part dans l'espace sud-slave, très largement majoritaire à l'échelle locale, fortement concentrée et adossée à son État-patrie, établie sur un territoire bien délimité et reconnu comme tel mais lourdement chargé d'histoire et disputé de longue date ; une minorité soumise de surcroît à un régime proprement discriminatoire et répressif, d'autant plus insupportable pour les intéressés qu'il succéda brutale- 
ment à quinze années de traitement particulièrement avantageux ; une minorité fortement mobilisée enfin, solidaire, organisée, rétive à toute idée de compromis et se définissant comme un peuple à part entière. Le Kosovo était de fait un cas extrême de polarisation : des positions diamétralement opposées, une intransigeance absolue de part et d'autre, une absence quasi-complète de communication, une propagande active des deux côtés et deux mondes faisant mine de s'ignorer, incarnés chacun par des institutions séparées dont l'autre récusait toute légitimité. Bref, tous les clignotants étaient au rouge. Or, qu'un conflit soit prévisible ne le rend pas nécessairement plus facile à prévenir ; cela témoigne plutôt du caractère ostensiblement critique de la situation. De ce point de vue, l'échec rencontré au Kosovo fut retentissant de par ses conséquences, mais reste relatif eu égard à la difficulté de la tâche. Cela ne dispense pas de souligner l'inefficacité de l'approche adoptée et de chercher à en comprendre les causes.

En premier lieu, ce n'est certes pas faute d' " alerte rapide " ou d'informations " fiables et précoces " que la diplomatie préventive a échoué dans le cas du Kosovo. Les maigres initiatives prises en 1992-1993 attestent au contraire que l'ensemble des gouvernements de la planète étaient fort conscients du danger et redoutaient notamment l'éventualité que le régime serbe ne tombe à bras raccourcis sur la population albanaise de la province. L'échec rencontré malgré tout provient d'abord de la politique de l'autruche adoptée par les principaux États concernés en vertu de laquelle la question du Kosovo fut enterrée à trois reprises : à La Haye (1991), Londres (1992) et Dayton (1995). Les raisons en sont connues, qui découlent de l'opposition à l'indépendance du Kosovo, de la priorité donnée au règlement des conflits ouverts et du marchandage implicite conclu, par voie de conséquence, avec Slobodan Milošević. En un mot, les Occidentaux abandonnèrent la province au régime serbe en échange de son concours sur d'autres dossiers et le firent avec d'autant moins de scrupules que le Kosovo devait à leurs yeux demeurer sous la juridiction de Belgrade.

En second lieu, l'affirmation selon laquelle les Occidentaux traitaient de cette question comme d'un problème de droits de l'Homme plutôt qu'un problème politique paraît erronée. Il est certain que de leur point de vue, les Albanais constituaient une minorité nationale et ne pouvaient à ce titre revendiquer le droit à l'autodétermination. Ce n'est pas dire qu'ils négligèrent le fond de l'affaire. Au contraire, c'est précisément parce qu'ils récusaient les aspirations à l'indépendance des Albanais qu'ils fermèrent les yeux, à partir de 1993 notamment, sur le sort infligé à ces derniers. Et par la suite, ils insistèrent bien davantage sur le règlement de la question du statut qu'ils n'exigèrent de Belgrade de mettre immédiatement un terme à l'oppression subie par la population albanaise. En conséquence, ils préconisèrent une solution toute trouvée - l'autonomie substantielle - au détriment du processus susceptible d'y aboutir. Si, à l'inverse, les Occidentaux avaient véritablement fait des droits de 
l'Homme leur priorité, ils auraient pu, et dû, œuvrer d'abord à l'amélioration du contexte général avant d'envisager le règlement du problème politique.

En troisième lieu, la question fut posée lors du déclenchement de l'intervention militaire de l'OTAN si toutes les options diplomatiques avaient bien été explorées. Il est clair qu'entre 1992 et 1998, les Occidentaux ne fournirent pas le maximum d'efforts pour tenter de débloquer la situation, loin s'en faut. Ils n'auraient peut-être pas été rendus à une telle extrémité au printemps 1999 s'ils s'étaient montrés auparavant plus actifs, plus audacieux, plus déterminés. Cependant, au cours des sept années qui précédèrent la campagne de bombardements, les Occidentaux et d'autres gouvernements avec eux tentèrent malgré tout de s'immiscer au Kosovo par la seule voie diplomatique et se heurtèrent à l'obstruction systématique du régime serbe. Invariablement, Slobodan Milošević a sapé ou rejeté chacune des tentatives faites pour amorcer un dialogue sur la question du Kosovo : il a refusé de traiter de ce sujet à La Haye en 1991 et Dayton en 1995 ; il a expulsé à la première occasion la seule présence internationale déployée à titre préventif (une mission purement civile de vingt personnes qu'il n'avait d'ailleurs pas acceptée lui-même) ; il a fermé la porte aux différents médiateurs mandatés par les institutions internationales - Geert Ahrens (1993), Martin Lutz (1996), Max van der Stoel (1997) et Felipe Gonzales (1998). Aucune de ces initiatives n'était soutenue par la force ou la menace, aucune ne visait à l'instauration d'un protectorat international, aucune ne préfigurait le déploiement d'une présence militaire, aucune n'était promue par l'OTAN, aucune ne remettait en cause la souveraineté de la Serbie ou de la RFY, aucune ne préjugeait de l'indépendance du Kosovo - tout au contraire. Si donc les Occidentaux agirent de façon aléatoire et sans conviction, Slobodan Milošević fit, lui, obstacle avec un aplomb indéfectible à toutes les options qui lui furent présentées. En définitive, la question n'est pas de savoir si les possibilités diplomatiques furent bien épuisées, mais si celles-ci avaient jamais existé.

En ce sens, et en quatrième lieu, l'approche coopérative de la prévention qui fut adoptée dans le cas du Kosovo était marquée au sceau de l'inanité. De fait, les initiatives prises ne firent pas évoluer d'un pouce la situation sur le terrain. Il paraît clair à cet égard que le statu quo qui régna jusqu'en 1997 ne résultait pas de pressions extérieures contraignant les protagonistes à la retenue, mais des considérations tactiques de Belgrade d'une part et de la LDK de l'autre. Mais cette approche coopérative se doubla en outre de perversité en ce qu'elle conduisit à conforter Slobodan Milošević, laissé libre d'agir à sa guise au Kosovo, et à affaiblir Ibrahim Rugova, incapable quant à lui de faire valoir le moindre résultat. La LDK, naturellement, n'est pas exempte de tout reproche dans cette affaire, qui promettait la lune aux siens sans avoir les moyens de la décrocher et manifestait sur le fond une intransigeance qui n'avait pas grand chose à envier au régime serbe. Il reste que Slobodan Milošević usait et abusait 
de la force quand Ibrahim Rugova s'interdisait d'y recourir. Et qu'en matière de prévention des conflits armés, la non-violence, fut-elle une nécessité faite vertu, n'est pas une donnée anodine. La LDK avait sans doute pour défauts d'embrigader la population et d'entretenir ses rêves les plus absolus, mais pour avantages de la contrôler, de se conformer à une ligne pacifiste et d'éviter ainsi un bain de sang. Or, les Occidentaux ne la soutinrent pas. Ils firent part à Ibrahim Rugova de leur " estime " mais ne l'aidèrent naturellement pas à maintenir à flot les institutions parallèles qu'ils souhaitaient voir disparaitre à terme et dont la déliquescence progressive ajouta au discrédit de la LDK. Ils composèrent avec Slobodan Milošević sans jamais obtenir de lui la moindre concession sur la province, mais en concoctant un Accord de Dayton qui s'apparentait à une véritable invitation à la guerre. Puis ils laissèrent filer le temps en pratiquant la politique de l'autruche, ce qui finit par exacerber impatience et frustration au sein de la population albanaise. En fin de compte, les Occidentaux ont marginalisé les pacifistes et dégagé le terrain aux partisans de la violence. Ce n'était pas la meilleure façon de faire de la prévention. 